Document downloaded from:

http://hdl.handle.net/10251/172305

This paper must be cited as:

Manzano, A.; Rueda, P.; Sánchez Pérez, EA. (2020). Closed injective ideals of multilinear operators, related measures and interpolation. Mathematische Nachrichten. 293(3):510-532. https://doi.org/10.1002/mana.201800415

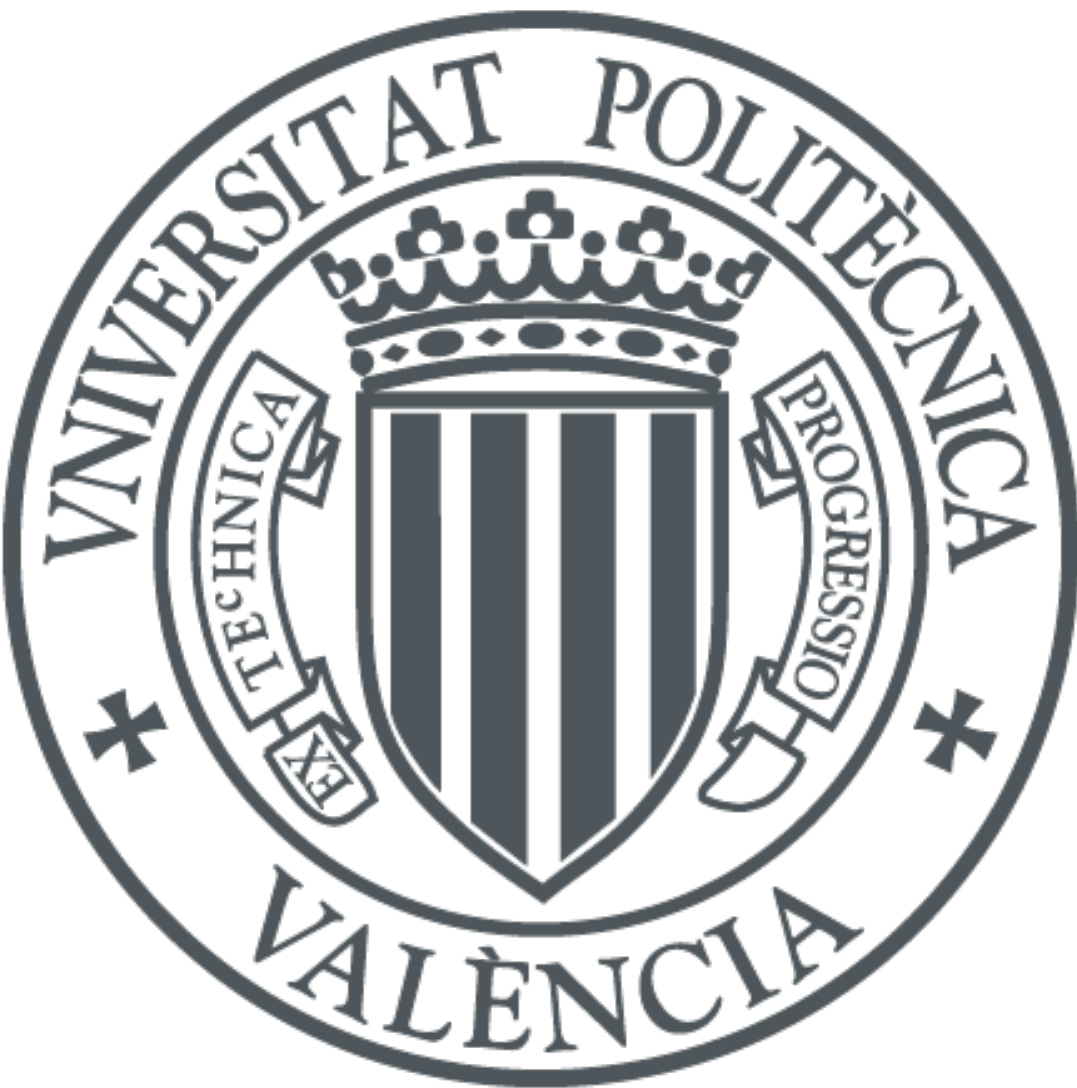

The final publication is available at

https://doi.org/10.1002/mana.201800415

Copyright John Wiley \& Sons

Additional Information

This is the peer reviewed version of the following article: Manzano, A, Rueda, P, SánchezPérez, EA. Closed injective ideals of multilinear operators, related measures and interpolation. Mathematische Nachrichten. 2020; 293: 510-532, which has been published in final form at https://doi.org/10.1002/mana.201800415. This article may be used for noncommercial purposes in accordance with Wiley Terms and Conditions for Self-Archiving. 


\title{
Closed injective ideals of multilinear operators, related measures and interpolation
}

February 13, 2021

\begin{abstract}
Antonio Manzano
Departamento de Matemáticas y Computación, Universidad de Burgos, Escuela Politécnica Superior, C/ Villadiego s/n, 09001 Burgos, Spain.

amanzano@ubu.es

Pilar Rueda

Departamento de Análisis Matemático, Universitat de València, Campus de

Burjassot, C/ Dr. Moliner 50, 46100 Burjassot (Valencia), Spain. pilar.rueda@uv.es

Enrique A. Sánchez-Pérez

Instituto Universitario de Matemática Pura y Aplicada, Universitat Politècnica de València, Camino de Vera s/n, 46022 Valencia, Spain. easancpe@mat.upv.es
\end{abstract}

\begin{abstract}
We introduce and discuss several ways of extending the inner measure arisen from the closed injective hull of an ideal of linear operators to the multilinear case. In particular, we consider new measures that allow to characterize the operators that belong to a closed injective ideal of multilinear operators as those having measure equal to zero. Some interpolation formulas for these measures, and consequently interpolation results involving ideals of multilinear operators, are established. Examples and applications related to summing multilinear operators are also shown.
\end{abstract}

keywords: Ideal of multilinear operators, closed ideal, injective ideal, measure associated to an ideal, interpolation

AMS Class. Primary: 47L22; Secondary: 46B70, 46G25

\section{Introduction}

A fruitful classical way of studying some properties of a linear operator is by considering functionals or measures (of the operator) related to operator ideals. An example of this is the inner measure $\beta_{\mathcal{I}}$ associated to an arbitrary ideal $\mathcal{I}$ of linear operators. We recall that for a continuous linear operator $T: E \rightarrow F$, 
$\beta_{\mathcal{I}}(T)=\beta_{\mathcal{I}}(T: E \rightarrow F):=\inf \{\varepsilon>0:$ there are a Banach space $Z$ and $R \in \mathcal{I}(E ; Z)$ such that $\|T x\|_{F} \leq \varepsilon\|x\|_{E}+\|R x\|_{Z}$, for any $\left.x \in E\right\}$. This measure was introduced by Tylli [34] in 1995 and determines the operators $T$ that belong to the closed injective hull $\overline{\mathcal{I}}^{\text {inj }}$ (i.e. the smallest closed injective ideal containing $\mathcal{I}$ ) of $\mathcal{I}$ as those for which $\beta_{\mathcal{I}}(T)=0$ (see [20, Theorem 20.7.3]). In particular when $\mathcal{I}$ is closed and injective, $T \in \mathcal{I}$ if and only if $\beta_{\mathcal{I}}(T)=0$. Therefore, the inner measure provides a way of characterizing when a given operator belongs to the closed injective hull of a linear operator ideal, and so it allows to quantify (in some sense) how far is the operator from such a hull.

As far as we know, there is no such notion in the literature as the inner measure in the setting of multilinear operators. In the present paper we introduce some functions that extend the inner measure to the multilinear case. The paper is organized in five sections. After the preliminary Sections 1 and 2, we introduce in Section 3 the definitions and main properties of two measures, naturally given and associated to an ideal of multilinear operators, that generalize the aforementioned measure defined by Tylli. In addition, in this section, we establish results concerning the closed injective hull of certain classes of ideals of multilinear operators. Section 4 is devoted to establish interpolation formulas for the new measures and to obtain certain consequences of them. Finally, we show in Section 5 examples and applications related to summing multilinear operators, using the Jarchow-Matter interpolation procedure (see [21]). This point of view of Jarchow and Matter [21] has turned out to be very useful in the study of new (and other well-known) ideals of linear operators and different properties of Banach spaces (see for example [23] and references therein). Other interpolation ideas also used in the multilinear setting giving succesfull results can be seen, for instance, in [10], [14] and [29].

\section{Preliminaries}

Throughout the paper we consider real or complex Banach spaces without distinction. If $E_{1}, \ldots, E_{n}$ and $F$ are Banach spaces, then $\mathcal{L}\left(E_{1}, \ldots, E_{n} ; F\right)$ stands for the Banach space of all continuous $n$-linear operators $T: E_{1} \times \cdots \times E_{n} \rightarrow F$ with the norm

$$
\|T\|:=\sup \left\{\left\|T\left(x_{1}, \ldots, x_{n}\right)\right\|_{F}: x_{1} \in B_{E_{1}}, \ldots, x_{n} \in B_{E_{n}}\right\},
$$

where $B_{E_{j}}$ is the closed unit ball of $E_{j}, j=1, \ldots, n$. In particular, $\mathcal{L}(E ; F)$ is the Banach space of all continuous linear operators from $E$ into $F$.

Let $E_{1} \otimes \cdots \otimes E_{n}$ denote the tensor product of $E_{1}, \ldots, E_{n}$ and let $\pi$ be the projective norm given by

$$
\pi(\theta):=\inf \sum_{j=1}^{m}\left\|x_{1}^{j}\right\| \cdots\left\|x_{n}^{j}\right\|, \quad \theta \in E_{1} \otimes \cdots \otimes E_{n},
$$

where the infimum is taken over all possible representations of $\theta$ of the form $\theta=\sum_{j=1}^{m} x_{1}^{j} \otimes \cdots \otimes x_{n}^{j}, x_{i}^{j} \in E_{i}(i=1, \ldots, n)$. The completed projective tensor 
product is denoted by $E_{1} \hat{\otimes}_{\pi} \cdots \hat{\otimes}_{\pi} E_{n}$.

Given $T \in \mathcal{L}\left(E_{1}, \ldots, E_{n} ; F\right), T_{L}$ stands for the linearization of $T$, that is, the unique continuous linear operator $T_{L}: E_{1} \hat{\otimes}_{\pi} \cdots \hat{\otimes}_{\pi} E_{n} \rightarrow F$ such that $T_{L}\left(x_{1} \otimes\right.$ $\left.\cdots \otimes x_{n}\right)=T\left(x_{1}, \ldots, x_{n}\right)$, for any $x_{1} \in E_{1}, \ldots, x_{n} \in E_{n}$.

The notion of linear operator ideal (see [30]) extends to multilinear operators as follows.

Let $n \in N$ be fixed. An ideal of $n$-linear operators, or an $n$-ideal, is a class $\mathcal{M}_{n}$ of $n$-linear maps such that for all Banach spaces $E_{1}, \ldots, E_{n}$ and $F$, the components $\mathcal{M}_{n}\left(E_{1}, \ldots, E_{n} ; F\right):=\mathcal{L}\left(E_{1}, \ldots, E_{n} ; F\right) \cap \mathcal{M}_{n}$ satisfy

(i) $\mathcal{M}_{n}\left(E_{1}, \ldots, E_{n} ; F\right)$ is a linear subspace of $\mathcal{L}\left(E_{1}, \ldots, E_{n} ; F\right)$ that contains the $n$-linear maps of finite type.

(ii) If $R \in \mathcal{L}(F ; H), T \in \mathcal{M}_{n}\left(E_{1}, \ldots, E_{n} ; F\right)$ and $S_{j} \in \mathcal{L}\left(G_{j} ; E_{j}\right)$, for $j=$ $1, \ldots, n$, then $R \circ T \circ\left(S_{1}, \ldots, S_{n}\right) \in \mathcal{M}_{n}\left(G_{1}, \ldots, G_{n} ; H\right)$.

If for each $n \in N, \mathcal{M}_{n}$ is an ideal of $n$-linear operators, the class

$$
\mathcal{M}:=\bigcup_{n=1}^{\infty} \mathcal{M}_{n}
$$

is called an ideal of multilinear operators or a multi-ideal.

The multi-ideal of all continuous multilinear operators is denoted by $\mathcal{L}$. Let us recall the construction of two examples of ideals of $n$-linear operators that can be found in [31] and are related to the classical notion of operator ideal [30]. To avoid confusions, we will use the letter $\mathcal{I}$ to denote an ideal of linear operators (instead of $\mathcal{M}_{1}$ or $\mathcal{I}_{1}$ ). Thus, a sequence as $\mathcal{I}_{1}, \ldots, \mathcal{I}_{n}$ means a sequence of $n$ ideals of linear operators.

On the other hand, throughout the paper the symbol $\stackrel{[i]}{\cdots}$. means that the $i$-th term, or the $i$-th coordinate, does not appear.

Linearization ideal. Let $\mathcal{I}_{1}, \ldots, \mathcal{I}_{n}$ be linear operator ideals. The ideal of $n$-linear operators $\left[\mathcal{I}_{1}, \ldots, \mathcal{I}_{n}\right]$ is defined as follows: Let $T \in \mathcal{L}\left(E_{1}, \ldots, E_{n} ; F\right)$,

$T \in\left[\mathcal{I}_{1}, \ldots, \mathcal{I}_{n}\right]\left(E_{1}, \ldots, E_{n} ; F\right)$ ifandonlyif $T_{i} \in \mathcal{I}_{i}\left(E_{i} ; \mathcal{L}\left(E_{1}, .{ }^{[i]} ., E_{n} ; F\right)\right), i=1,2, \ldots, n$, where $T_{i}: E_{i} \rightarrow \mathcal{L}\left(E_{1}, .{ }^{[i]} ., E_{n} ; F\right)$ is defined as

$$
T_{i}\left(x_{i}\right)\left(x_{1}, \cdot[\cdot], x_{n}\right):=T\left(x_{1}, \ldots, x_{n}\right), x_{1} \in E_{1}, \ldots, x_{n} \in E_{n} .
$$

Factorization ideal. Let $\mathcal{I}_{1}, \ldots, \mathcal{I}_{n}$ be linear operator ideals. The ideal of $n$-linear operators $\mathcal{L}\left(\mathcal{I}_{1}, \ldots, \mathcal{I}_{n}\right)$ is defined as follows: Let $T \in \mathcal{L}\left(E_{1}, \ldots, E_{n} ; F\right)$,

$T \in \mathcal{L}\left(\mathcal{I}_{1}, \ldots, \mathcal{I}_{n}\right)\left(E_{1}, \ldots, E_{n} ; F\right)$ ifandonlyifT factorsas $T=S \circ\left(R_{1}, \ldots, R_{n}\right)$, for some $R_{j} \in \mathcal{I}_{j}\left(E_{j} ; G_{j}\right)(j=1,2, \ldots, n)$ and $S \in \mathcal{L}\left(G_{1}, \ldots, G_{n} ; F\right)$.

Although both procedures (linearization and factorization) give, in general, different ideals of multilinear operators (see [10, p.741]), the inclusion 
$\mathcal{L}\left(\mathcal{I}_{1}, \ldots, \mathcal{I}_{n}\right) \subset\left[\mathcal{I}_{1}, \ldots, \mathcal{I}_{n}\right]$ always holds. However, there are examples of ideals for which the inclusion becomes an equality. For instance, if $\mathcal{I}_{i}$ is the ideal of compact operators $\mathcal{K}$ for $i=1,2, \ldots, n$, or if $\mathcal{I}_{i}$ is the ideal of weakly compact operators $\mathcal{W}$ for $i=1, \ldots, n$, then $\mathcal{L}\left(\mathcal{I}_{1}, \ldots, \mathcal{I}_{n}\right)=\left[\mathcal{I}_{1}, \ldots, \mathcal{I}_{n}\right]$. This is not a mère coincidence: the ideals of compact and weakly compact operators share the properties of being closed and injective. González and Gutiérrez proved that both procedures (linearization and factorization) coincide when they are applied to a single closed injective operator ideal $\mathcal{I}_{1}=\cdots=\mathcal{I}_{n}=\mathcal{I}$ (see $[16$, Theorem 4] and [17]). More recently Braunss and Junek [10, Theorem 3.4] have shown that $\mathcal{L}\left(\mathcal{I}_{1}, \ldots, \mathcal{I}_{n}\right)=\left[\mathcal{I}_{1}, \ldots, \mathcal{I}_{n}\right]$ holds for different closed injective operator ideals $\mathcal{I}_{1}, \ldots, \mathcal{I}_{n}$. Under such a hypothesis the ideals of $n$-linear operators $\mathcal{L}\left(\mathcal{I}_{1}, \ldots, \mathcal{I}_{n}\right)$ and $\left[\mathcal{I}_{1}, \ldots, \mathcal{I}_{n}\right]$ turn out to be both closed and injective as well.

Let $\mathcal{M}_{n}$ be an ideal of $n$-linear operators. It will be denoted by $\overline{\mathcal{M}_{n}}$ the class of $n$-linear operators formed by components $\overline{\mathcal{M}_{n}}\left(E_{1}, \ldots, E_{n} ; F\right)$ that are given by the closure of $\mathcal{M}_{n}\left(E_{1}, \ldots, E_{n} ; F\right)$ in $\mathcal{L}\left(E_{1}, \ldots, E_{n} ; F\right) . \mathcal{M}_{n}$ is said to be closed when $\overline{\mathcal{M}_{n}}=\mathcal{M}_{n}$.

The injective hull $\mathcal{M}_{n}^{\text {inj }}$ of $\mathcal{M}_{n}$ is defined as follows: $T \in \mathcal{L}\left(E_{1}, \ldots, E_{n} ; F\right)$ belongs to $\mathcal{M}_{n}^{i n j}\left(E_{1}, \ldots, E_{n} ; F\right)$ if $J_{F} \circ T \in \mathcal{M}_{n}\left(E_{1}, \ldots, E_{n} ; \ell_{\infty}\left(B_{F^{*}}\right)\right)$, where $J_{F}: F \rightarrow \ell_{\infty}\left(B_{F^{*}}\right)$ is the natural metric injection given by $J_{F}(y)=\left(\left\langle y, y^{*}\right\rangle\right)_{y^{*} \in B_{F^{*}}}$. $\mathcal{M}_{n}$ is called injective if $\mathcal{M}_{n}=\mathcal{M}_{n}^{i n j}$, i.e. if for any Banach spaces $E_{1}, \ldots, E_{n}, F$ and each $n$-linear operator $T \in \mathcal{L}\left(E_{1}, \ldots, E_{n} ; F\right)$, it holds that $T \in \mathcal{M}_{n}\left(E_{1}, \ldots, E_{n} ; F\right)$ whenever $J_{F} \circ T \in \mathcal{M}_{n}\left(E_{1}, \ldots, E_{n} ; \ell_{\infty}\left(B_{F^{*}}\right)\right)$.

The closed injective hull $\overline{\mathcal{I}}^{i n j}$ of an ideal $\mathcal{I}$ of linear operators can be characterized as follows (see [20, Theorem 20.7.3(i)] or [21, Section 1,(2)(a)]): Take $T \in \mathcal{L}(E ; F)$, then $T \in \overline{\mathcal{I}}^{i n j}(E ; F)$ if and only if for each $\varepsilon>0$ there are a Banach space $Z$ and an operator $R \in \mathcal{I}(E ; Z)$ such that $\|T x\|_{F} \leq \varepsilon\|x\|_{E}+\|R x\|_{Z}$, for all $x \in E$.

Hence, as it was said in Introduction, the inner measure $\beta_{\mathcal{I}}$ of $T \in \mathcal{L}(E ; F)$, given by $\beta_{\mathcal{I}}(T)=\beta_{\mathcal{I}}(T: E \rightarrow F):=\inf \{\varepsilon>0:$ there are a Banach space $Z$ and $R \in \mathcal{I}(E ; Z)$ such that $\|T x\|_{F} \leq \varepsilon\|x\|_{E}+\|R x\|_{Z}$, for any $\left.x \in E\right\}$, satisfies that $\beta_{\mathcal{I}}(T)=$ $0 \Longleftrightarrow T \in \overline{\mathcal{I}}^{i n j}(E ; F)$. Therefore, $\beta_{\mathcal{I}}(T)=0$ if and only if $T \in \mathcal{I}(E ; F)$ when $\mathcal{I}$ is closed and injective.

\section{Measures associated to ideals of multilinear operators}

It is natural to investigate if it is possible to generalize the notion of inner measure to the setting of ideals of multilinear operators. We will deal with this issue in this section.

We start by stating two lemmas on the injective hull and the closed hull of an $n$-ideal $\left[\mathcal{I}_{1}, \ldots, \mathcal{I}_{n}\right]$ that are known results (see $[8$, p.309]). Because this type of $n$-ideal will play an important role in this paper, and for the sake of completeness, we include such lemmas and their proofs. First recall that a Banach space $H$ is said to be injective, or it has the metric extension property, 
if for any Banach space $G$, any closed linear subspace $E$ of $G$, and any $R \in$ $\mathcal{L}(E ; H)$, there exists an extension $S \in \mathcal{L}(G ; H)$ of $R$ with $\|S\|=\|R\|$. Let $\mathcal{I}_{1}, \ldots, \mathcal{I}_{n}$ be linear operator ideals. Then,

(a) $\left[\mathcal{I}_{1}, \ldots, \mathcal{I}_{n}\right]^{i n j} \subset\left[\mathcal{I}_{1}^{i n j}, \ldots, \mathcal{I}_{n}^{i n j}\right]$.

(b) $\overline{\left[\mathcal{I}_{1}, \ldots, \mathcal{I}_{n}\right]} \subset\left[\overline{\mathcal{I}_{1}}, \ldots, \overline{\mathcal{I}_{n}}\right]$.

(a) Take $T \in\left[\mathcal{I}_{1}, \cdots, \mathcal{I}_{n}\right]^{i n j}\left(E_{1}, \ldots, E_{n} ; F\right)$. For each $i=1, \ldots, n$, consider the mapping

$$
j_{i}: \mathcal{L}\left(E_{1}, .{ }^{[i]} ., E_{n} ; F\right) \rightarrow \mathcal{L}\left(E_{1}, .\left[{ }^{i}\right], E_{n} ; \ell_{\infty}\left(B_{F^{*}}\right)\right)
$$

given by $j_{i}(A):=J_{F} \circ A, A \in \mathcal{L}\left(E_{1},{ }^{[i]} ., E_{n} ; F\right)$. Since $J_{F}$ is an isometry, the map $j_{i}$ is a metric injection. For any $x_{i} \in E_{i}$, we have $\mathrm{j}_{i}\left(T_{i}\left(x_{i}\right)\right)\left(x_{1},{ }^{[i]}, x_{n}\right)=$ $\left(J_{F} \circ\left(T_{i}\left(x_{i}\right)\right)\right)\left(x_{1}, .[i] ., x_{n}\right)$

$=J_{F}\left(T\left(x_{1}, \ldots, x_{n}\right)\right)=\left(J_{F} \circ T\right)_{i}\left(x_{i}\right)\left(x_{1}, .\left[{ }^{[i]}, x_{n}\right)\right.$. Using the metric extension property of $\ell_{\infty}\left(B_{\mathcal{L}\left(E_{1},[i], E_{n} ; F\right)^{*}}\right)$, there is a continuous linear mapping

$$
\phi_{i}: \mathcal{L}\left(E_{1},{ }^{[i]} ., E_{n} ; \ell_{\infty}\left(B_{F^{*}}\right)\right) \rightarrow \ell_{\infty}\left(B_{\mathcal{L}\left(E_{1},[i], E_{n} ; F\right)^{*}}\right)
$$

such that $\phi_{i} \circ j_{i}=J_{\mathcal{L}\left(E_{1},[i], E_{n} ; F\right)}$. Then,

$J_{\mathcal{L}\left(E_{1},[i], E_{n} ; F\right)} \circ T_{i}=\phi_{i} \circ\left(J_{F} \circ T\right)_{i} \in \mathcal{I}_{i}\left(E_{i} ; \mathcal{L}\left(E_{1},{ }^{[i]} ., E_{n} ; \ell_{\infty}\left(B_{\mathcal{L}\left(E_{1},[i], E_{n} ; F\right)^{*}}\right)\right)\right.$.

Hence, $T_{i} \in \mathcal{I}_{i}^{i n j}\left(E_{i} ; \mathcal{L}\left(E_{1},,{ }^{[i]} \cdot, E_{n} ; F\right)\right)$ for all $i=1, \ldots, n$. Thus, $T \in\left[\mathcal{I}_{1}^{i n j}, \cdots, \mathcal{I}_{n}^{i n j}\right]$.

(b) Let $T \in \overline{\left[\mathcal{I}_{1}, \ldots, \mathcal{I}_{n}\right]}\left(E_{1}, \ldots, E_{n} ; F\right)$. Given $\varepsilon>0$, we find $n$-linear operators $A, B \in \mathcal{L}\left(E_{1}, \ldots, E_{n} ; F\right)$ such that $A \in\left[\mathcal{I}_{1}, \ldots, \mathcal{I}_{n}\right]\left(E_{1}, \ldots, E_{n} ; F\right)$, $\|B\|<\varepsilon$ and $T=A+B$. For each $i=1, \ldots, n$, we have $T_{i}=A_{i}+B_{i}, A_{i} \in$ $\mathcal{I}_{i}\left(E_{i} ; \mathcal{L}\left(E_{1},[i] ., E_{n} ; F\right)\right)$ and $\left\|B_{i}\right\|<\varepsilon$. Then, $\left.T_{i} \in \overline{\mathcal{I}_{i}}\left(E_{i} ; \mathcal{L}\left(E_{1} \quad . i\right] ., E_{n} ; F\right)\right)$. Hence, $T \in\left[\overline{\mathcal{I}_{1}}, \ldots, \overline{\mathcal{I}_{n}}\right]$.

As a direct consequence of Lemma 3 we derive the following result.

Let $\mathcal{I}_{1}, \ldots, \mathcal{I}_{n}$ be linear operator ideals.

(a) If $\mathcal{I}_{1}, \ldots, \mathcal{I}_{n}$ are injective, then $\left[\mathcal{I}_{1}, \ldots, \mathcal{I}_{n}\right]$ is injective too.

(b) If $\mathcal{I}_{1}, \ldots, \mathcal{I}_{n}$ are closed, then $\left[\mathcal{I}_{1}, \ldots, \mathcal{I}_{n}\right]$ is closed too.

(c) If $\mathcal{I}_{i}=\overline{\mathcal{I}}_{i}^{i n j}, i=1, \ldots, n$, then $\left[\mathcal{I}_{1}, \ldots, \mathcal{I}_{n}\right]={\overline{\left[\mathcal{I}_{1}, \ldots, \mathcal{I}_{n}\right]}}^{i n j}$.

(a) It follows from

$$
\left[\mathcal{I}_{1}, \ldots, \mathcal{I}_{n}\right]^{i n j} \subset\left[\mathcal{I}_{1}^{i n j}, \ldots, \mathcal{I}_{n}{ }^{i n j}\right]=\left[\mathcal{I}_{1}, \ldots, \mathcal{I}_{n}\right] .
$$

(b) The next inclusion gives the result:

$$
\overline{\left[\mathcal{I}_{1}, \ldots, \mathcal{I}_{n}\right]} \subset\left[\overline{\mathcal{I}_{1}}, \ldots, \overline{\mathcal{I}_{n}}\right]=\left[\mathcal{I}_{1}, \ldots, \mathcal{I}_{n}\right] .
$$


Now (c) is obvious.

Let $\mathcal{I}_{1}, \ldots, \mathcal{I}_{n}$ be linear operator ideals. By $[8$, p.309] we trivially have

$$
{\overline{\mathcal{L}}\left(\overline{\mathcal{I}}_{1}, \ldots, \overline{\mathcal{I}}_{n}\right)}^{i n j} \subset \mathcal{L}\left(\overline{\mathcal{I}}^{i n j}, \ldots,{\overline{\mathcal{I}_{n}}}^{i n j}\right) .
$$

Let us show that for some particular operators the other inclusion also holds. To prove the next result, we need to extend a continuous multilinear operator $S: E_{1} \times \cdots \times E_{n} \rightarrow F$ to some continuous multilinear operator $\operatorname{ext}(S): E_{1}^{* *} \times \cdots \times E_{n}^{* *} \rightarrow F^{* *}$. Continuous bilinear operators $A: E_{1} \times E_{2} \rightarrow F$ were extended to continuous bilinear operator from $E_{1}^{* *} \times E_{2}^{* *}$ into $F^{* *}$ by Arens [1]. This extension is built by considering three times in a row the following transpose: $\quad A^{t}: F^{*} \times E_{1} \rightarrow E_{2}^{*}$

$$
\left(y^{*}, x_{1}\right) \quad A^{t}\left(y^{*}, x_{1}\right)\left(x_{2}\right)=y^{*}\left(A\left(x_{1}, x_{2}\right)\right),
$$

$x_{1} \in E_{1}, x_{2} \in E_{2}$ and $y^{*} \in F^{*}$. This procedure gives two, in general different, extensions: $A^{t t t}$ and $A^{T t t t T}$, where $B^{T}\left(x_{1}, x_{2}\right)=B\left(x_{2}, x_{1}\right)$ for any bilinear mapping $B$, and are known as Arens products. This procedure was generalized by Aron and Berner [2] to arbitrary multilinear mappings. Given a continuous multilinear operator $S: E_{1} \times \cdots \times E_{n} \rightarrow F$ we will denote $A B(S): E_{1}^{* *} \times \cdots \times E_{n}^{* *} \rightarrow F^{* *}$ one of the Aron and Berner extensions of $S$.

Let $\mathcal{I}_{1}, \ldots, \mathcal{I}_{n}$ be linear operator ideals and let $E_{1}, \ldots, E_{n}, F$ be Banach spaces. If $S \in \mathcal{L}\left(c_{0}, \ldots, c_{0} ; F\right)$ and $R_{i} \in \overline{\mathcal{I}}_{i}^{i n j}\left(E_{i} ; c_{0}\right)$ for each $i=1, \ldots, n$, then $S \circ\left(R_{1}, \ldots, R_{n}\right) \in \overline{\mathcal{L}\left(\mathcal{I}_{1}, \ldots, \mathcal{I}_{n}\right)}{ }^{i n j}$.

We can trivially assume $S \neq 0$. Fix $\varepsilon>0$ and $i \in\{1, \ldots, n\}$. Since $R_{i} \in \overline{\mathcal{I}}_{i}^{i n j}\left(E_{i} ; c_{0}\right)$, there exist continuous linear operators $A_{i}, B_{i} \in \mathcal{L}\left(E_{i} ; c_{0}\right)$ such that

$$
A_{i} \in \mathcal{I}_{i}^{i n j}\left(E_{i} ; c_{0}\right),\left\|B_{i}\right\|<\frac{\varepsilon^{1 / n}}{\|S\|^{1 / n}} \text { and } R_{i}=A_{i}+B_{i} .
$$

Let $\left\{e_{j}: j \in N\right\}$ be the usual canonical basis in $\ell_{1}$. Having in mind that $\left(c_{0}^{*}\right)^{*}=\left(\ell_{1}\right)^{*}=\ell_{\infty}$, define the map

$$
P: \ell_{\infty}\left(B_{\ell_{1}}\right) \rightarrow \ell_{\infty}, \quad P(\eta)=\left(\eta_{e_{j}}\right)_{j=1}^{\infty},
$$

for any $\eta:=\left(\eta_{y^{*}}\right)_{y^{*} \in B_{\ell_{1}}} \in \ell_{\infty}\left(B_{\ell_{1}}\right)$. Clearly the map $P$ is well-defined, linear and continuous, with $\|P\| \leq 1$. Moreover, $P \circ J_{c_{0}}=I_{c_{0}}$, where $I_{c_{0}}: c_{0} \rightarrow \ell_{\infty}$ is the canonical injection.

Take any of the Aron and Berner extensions of $S: c_{0} \times \cdots \times c_{0} \rightarrow F$, denoted by $A B(S): \ell_{\infty} \times \cdots \times \ell_{\infty} \rightarrow F^{* *}$. Consider the canonical isometric inclusions $I_{F}: F \rightarrow F^{* *}$ and $K_{F}: F^{* *} \rightarrow \ell_{\infty}\left(B_{F^{*}}\right)$, given by $y^{* *} \in F^{* *} \rightarrow$ $\left(\left\langle y^{*}, y^{* *}\right\rangle\right)_{y^{*} \in B_{F^{*}}}$. These mappings are related via the equality $J_{F}=K_{F} \circ I_{F}$. Since $J_{c_{0}} \circ A_{i} \in \mathcal{I}_{i}\left(E_{i} ; \ell_{\infty}\left(B_{\ell_{1}}\right)\right)$ for an arbitrary $i \in\{1, \ldots, n\}$, it follows that the map

$$
T_{0}:=K_{F} \circ A B(S) \circ\left(P \circ J_{c_{0}} \circ A_{1}, \ldots, P \circ J_{c_{0}} \circ A_{n}\right)
$$

belongs to $\mathcal{L}\left(\mathcal{I}_{1}, \ldots, \mathcal{I}_{n}\right)\left(E_{1}, \ldots, E_{n} ; \ell_{\infty}\left(B_{F^{*}}\right)\right)$. Besides,

$$
T_{0}=K_{F} \circ A B(S) \circ\left(I_{c_{0}} \circ A_{1}, \ldots, I_{c_{0}} \circ A_{n}\right)=J_{F} \circ S \circ\left(A_{1}, \ldots, A_{n}\right) .
$$


Then, $S \circ\left(A_{1}, \ldots, A_{n}\right) \in \mathcal{L}\left(\mathcal{I}_{1}, \ldots, \mathcal{I}_{n}\right)^{i n j}\left(E_{1}, \ldots, E_{n} ; F\right)$. Since $\| S \circ\left(R_{1}, \ldots, R_{n}\right)-$ $S \circ\left(A_{1}, \ldots, A_{n}\right)\|\leq\| S\|\| R_{1}-A_{1}\|\cdots\| R_{n}-A_{n} \|$

$=\|S\|\left\|B_{1}\right\| \cdots\left\|B_{n}\right\| \leq\|S\| \frac{\varepsilon}{\|S\|}=\varepsilon$, we conclude that $S \circ\left(R_{1}, \ldots, R_{n}\right) \in$ ${\overline{\mathcal{L}\left(\mathcal{I}_{1}, \ldots, \mathcal{I}_{n}\right)}}^{i n j}\left(E_{1}, \ldots, E_{n} ; F\right)$.

Let us denote by $\mathcal{L}_{c_{0}}\left(\mathcal{I}_{1}, \ldots, \mathcal{I}_{n}\right)$ those elements in $\mathcal{L}\left(\mathcal{I}_{1}, \ldots, \mathcal{I}_{n}\right)$ that factor through $c_{0} \times \cdots \times c_{0}$, i.e. $T \in \mathcal{L}_{c_{0}}\left(\mathcal{I}_{1}, \ldots, \mathcal{I}_{n}\right)\left(E_{1}, \ldots, E_{n} ; F\right)$ if $T=$ $S \circ\left(R_{1}, \ldots, R_{n}\right)$ for some $S \in \mathcal{L}\left(c_{0}, \ldots, c_{0} ; F\right)$ and some $R_{i} \in \mathcal{I}_{i}\left(E_{i} ; c_{0}\right), i=$ $1, \ldots, n$. Then, Theorem 3 can be rephrased as follows:

$$
\mathcal{L}_{c_{0}}\left(\overline{\mathcal{I}}^{i n j}, \ldots,{\overline{\mathcal{I}_{n}}}^{i n j}\right) \subset{\overline{\mathcal{L}\left(\mathcal{I}_{1}, \ldots, \mathcal{I}_{n}\right)}}^{i n j} .
$$

Next let us extend the inner measure introduced by Tylli [34] to the setting of multilinear operators. Let $\mathcal{I}_{1}, \ldots, \mathcal{I}_{n}$ be linear operator ideals. For $T \in \mathcal{L}\left(E_{1}, \ldots, E_{n} ; F\right), \quad \beta_{\left[\mathcal{I}_{1}, \ldots, \mathcal{I}_{n}\right]}(T)=\beta_{\left[\mathcal{I}_{1}, \ldots, \mathcal{I}_{n}\right]}\left(T: E_{1} \times \cdots \times E_{n} \rightarrow F\right):=$ $\inf \left\{\varepsilon>0:\right.$ thereareBanachspaces $\mathrm{Z}_{i}$ and $\mathrm{R}_{i} \in \mathcal{I}_{i}\left(E_{i} ; Z_{i}\right)$ sothatif $x_{1} \in E_{1}, \ldots x_{n} \in$ $E_{n}$

$\left.\left\|T\left(x_{1}, \ldots, x_{n}\right)\right\|_{F} \leq \varepsilon\left\|x_{1}\right\| \cdots\left\|x_{n}\right\|+\min _{i \in\{1, \ldots, n\}}\left\{\left\|R_{i}\left(x_{i}\right)\right\|\left\|x_{1}\right\| \stackrel{[i]}{\cdot \cdots}\left\|x_{n}\right\|\right\}\right\}$.

The following result generalizes the well-known characterization of a closed injective linear operator ideal (that can be found in [20, Theorem 20.7.3(i)]). Let $\mathcal{I}_{1}, \ldots, \mathcal{I}_{n}$ be linear operator ideals and let $T \in \mathcal{L}\left(E_{1}, \ldots, E_{n} ; F\right)$. The following statements are equivalent.

(a) $T \in\left[\overline{\mathcal{I}}^{i n j}, \ldots, \overline{\mathcal{I}}_{n}^{i n j}\right]\left(E_{1}, \ldots, E_{n} ; F\right)$.

(b) For every $\varepsilon>0$ there are Banach spaces $Z_{i}$ and operators $R_{i} \in \mathcal{I}_{i}\left(E_{i} ; Z_{i}\right)$, $i=1, \ldots, n$, such that for all $x_{1} \in E_{1}, \ldots x_{n} \in E_{n}$

$$
\left\|T\left(x_{1}, \ldots, x_{n}\right)\right\|_{F} \leq \varepsilon\left\|x_{1}\right\| \cdots\left\|x_{n}\right\|+\min _{i \in\{1, \ldots, n\}}\left\{\left\|R_{i}\left(x_{i}\right)\right\|\left\|x_{1}\right\| \stackrel{[i]}{\cdots}\left\|x_{n}\right\|\right\} .
$$

(c) $\beta_{\left[\mathcal{I}_{1}, \ldots, \mathcal{I}_{n}\right]}(T)=0$.

If (a) holds, for every $\varepsilon>0$ there exists a Banach space $Z_{1}$ and an operator $R_{1} \in \mathcal{I}_{1}\left(E_{1} ; Z_{1}\right)$ such that for any $x_{1} \in E_{1}, \ldots, x_{n} \in E_{n}$

$\left\|T\left(x_{1}, \ldots, x_{n}\right)\right\|_{F}=\left\|T_{1}\left(x_{1}\right)\left(x_{2}, \ldots, x_{n}\right)\right\|_{F} \leq \varepsilon\left\|x_{1}\right\| \cdots\left\|x_{n}\right\|+\left\|R_{1}\left(x_{1}\right)\right\|\left\|x_{2}\right\| \cdots\left\|x_{n}\right\|$.

Since this also holds for every $i=2, \ldots, n$, we get

$$
\left\|T\left(x_{1}, \ldots, x_{n}\right)\right\|_{F} \leq \varepsilon\left\|x_{1}\right\| \cdots\left\|x_{n}\right\|+\min _{i \in\{1, \ldots, n\}}\left\{\left\|R_{i}\left(x_{i}\right)\right\|\left\|x_{1}\right\| \stackrel{[i]}{\cdots}\left\|x_{n}\right\|\right\},
$$

for each $x_{1} \in E_{1}, \cdots, x_{n} \in E_{n}$, that is, we obtain (b).

Now assume (b) and let us prove (a). Fix $\varepsilon>0$, then for $x_{1} \in E_{1}, \ldots, x_{n} \in$ $E_{n}$, we have $\left\|T\left(x_{1}, \ldots, x_{n}\right)\right\|_{F} \leq \varepsilon\left\|x_{1}\right\| \cdots\left\|x_{n}\right\|+\left\|R_{1}\left(x_{1}\right)\right\|\left\|x_{2}\right\| \cdots\left\|x_{n}\right\|$ $=\left(\varepsilon\left\|x_{1}\right\|+\left\|R_{1}\left(x_{1}\right)\right\|\right)\left\|x_{2}\right\| \cdots\left\|x_{n}\right\|$. Thus

$$
\left\|T_{1}\left(x_{1}\right)\right\|_{\mathcal{L}\left(E_{2}, \ldots, E_{n} ; F\right)}=\sup _{x_{2} \in B_{E_{2}}, \ldots, x_{n} \in B_{E_{n}}}\left\|T_{1}\left(x_{1}\right)\left(x_{2}, \ldots, x_{n}\right)\right\|_{F} \leq \varepsilon\left\|x_{1}\right\|+\left\|R_{1}\left(x_{1}\right)\right\| .
$$


Hence, $T_{1} \in{\overline{\mathcal{I}_{1}}}^{i n j}\left(E_{1} ; \mathcal{L}\left(E_{2}, \ldots, E_{n} ; F\right)\right)$. Reasoning similarly for $i=2, \ldots, n$, we can conclude that $T_{i} \in \overline{\mathcal{I}}_{i}^{i n j}\left(E_{i} ; \mathcal{L}\left(E_{1},{ }^{[i]}, ., E_{n} ; F\right)\right)$ for all $i=1, \ldots, n$. Then, it holds that $T \in\left[{\overline{\mathcal{I}_{1}}}^{i n j}, \ldots,{\overline{\mathcal{I}_{n}}}^{i n j}\right]\left(E_{1}, \ldots, E_{n} ; F\right)$, so we get (a).

It is obvious that (b) $\Longleftrightarrow$ (c).

Observe that the proof of Theorem 3 also allows to ensure that for any $T \in \mathcal{L}\left(E_{1}, \ldots, E_{n} ; F\right)$,

$$
\beta_{\left[\mathcal{I}_{1}, \ldots, \mathcal{I}_{n}\right]}(T)=\max \left\{\beta_{\mathcal{I}_{i}}\left(T_{i}\right): i=1, \ldots n\right\} .
$$

We have established in Theorem 3 that $\beta_{\left[\mathcal{I}_{1}, \ldots, \mathcal{I}_{n}\right]}$ just characterizes when an $n$-linear operator belongs to the ideal $\left[\overline{\mathcal{I}}_{1}^{i n j}, \ldots, \overline{\mathcal{I}}_{n}{ }^{i n j}\right]$. However, note that the inclusion $\left[{\overline{\left.\mathcal{I}_{1}, \ldots, \mathcal{I}_{n}\right]}}^{i n j} \subset\left[{\overline{\mathcal{I}_{1}}}^{i n j}, \ldots,{\overline{\mathcal{I}_{n}}}^{i n j}\right]\right.$ always holds, but it is not an equality in general. In fact, for $\mathcal{I}_{1}=\mathcal{I}_{2}=\mathcal{A}$, the ideal of (linear) approximable operators, it holds that $\overline{[\mathcal{A}, \mathcal{A}]}{ }^{i n j} \neq\left[\overline{\mathcal{A}}^{i n j}, \overline{\mathcal{A}}^{i n j}\right]$. It is well-known that $\overline{\mathcal{A}}^{i n j}=\mathcal{K}$ (see [30, Proposition 4.2.5, Remarks 4.6.13 and 4.7.13]). By [8, Example 3.4] (see also [3, proof of Theorem 4.5]), there exists a Banach space $E$ without the approximation property and an operator $u \in \mathcal{L}\left(E ; E^{*}\right)$ that is compact, symmetric and non-approximable. Let $A$ be the bilinear form on $E \times E$ considered in [8, Example 3.4], defined by $A(x, y)=u(x)(y)$. It is immediate that $A \in\left[\overline{\mathcal{A}}^{i n j}, \overline{\mathcal{A}}^{i n j}\right]$ since $A_{1}=A_{2}=u \in \mathcal{K}\left(E ; E^{*}\right)$. Nevertheless, if $A \in \overline{[\mathcal{A}, \mathcal{A}}^{i n j}(E, E ; F)$, taking into account that $F$ is an injective space and $\mathcal{A}$ is closed, it would follow (see [8, Corollary 2.6]) that

$$
A \in \overline{[\mathcal{A}, \mathcal{A}]}^{i n j}(E, E ; F)=\overline{[\mathcal{A}, \mathcal{A}]}(E, E ; F)=[\mathcal{A}, \mathcal{A}](E, E ; F),
$$

but this is a contradiction because $A_{1}=A_{2}=u \notin \mathcal{A}\left(E ; E^{*}\right)$.

In order to establish the next results, we recall that if $\mathcal{I}$ is a Banach linear operator ideal (see definition for example in [20, 19.3] or [15, Chapter I, Section $9]$ ), then the closed injective hull of $\mathcal{I}$ can be characterized as follows (see $[20$, Theorem 20.7.3(ii)] or [21, Section 1,(3)(a)]): An operator $T \in \mathcal{L}(E ; F)$ belongs to $\overline{\mathcal{I}}^{i n j}(E ; F)$ if and only if there are a function $N: R^{+} \rightarrow R^{+}$, a Banach space $G$ and an operator $S \in \mathcal{I}(E ; G)$ such that

$$
\|T(x)\|_{F} \leq N(\varepsilon)\|S(x)\|_{G}+\varepsilon\|x\|_{E}, \text { forevery }
$$

$\varepsilon>0$ and each $x \in E$. (1)

Let us see how the inequality (1) results in a general factorization theorem. We will proceed with the multilinear case directly.

Let $\mathcal{I}_{1}, \ldots, \mathcal{I}_{n}$ be Banach linear operator ideals, let $E_{1}, \ldots, E_{n}$ be Banach spaces and let $T \in \mathcal{L}\left(E_{1}, \ldots, E_{n} ; F\right)$. Then, $T \in\left[\overline{\mathcal{I}}_{1}^{i n j}, \ldots, \overline{\mathcal{I}}_{n}{ }^{i n j}\right]\left(E_{1}, \ldots, E_{n} ; F\right)$ if and only if for each $i=1, \ldots, n$, there exist a function $N_{i}: R^{+} \rightarrow R^{+}$and a 
linear operator $S_{i} \in \mathcal{I}_{i}\left(E_{i} ; G_{i}\right)$ such that

$$
\left\|T\left(x_{1}, \ldots, x_{n}\right)\right\| \leq\left(N_{1}\left(\varepsilon_{1}\right)\left\|S_{1}\left(x_{1}\right)\right\|+\varepsilon_{1}\left\|x_{1}\right\|\right) \cdots\left(N_{n}\left(\varepsilon_{n}\right)\left\|S_{n}\left(x_{n}\right)\right\|+\varepsilon_{n}\left\|x_{n}\right\|\right),
$$

for all $\varepsilon_{1}>0, \ldots, \varepsilon_{n}>0$ and $x_{1} \in E_{1}, \ldots, x_{n} \in E_{n}$.

Assume first that $T \in\left[\overline{\mathcal{I}}_{1}^{i n j}, \ldots, \overline{\mathcal{I}}_{n}{ }^{i n j}\right]\left(E_{1}, \ldots, E_{n} ; F\right)$. Then, there are Banach spaces $H_{1}, \ldots, H_{n}$, linear operators $R_{i} \in \overline{\mathcal{I}}_{i}^{i n j}\left(E_{i} ; H_{i}\right)$ and a continuous $n$-linear operator $S \in \mathcal{L}\left(H_{1}, \ldots, H_{n} ; F\right)$ such that $T=S \circ\left(R_{1}, \ldots, R_{n}\right)$. By (1), there are functions $N_{i}: R^{+} \rightarrow R^{+}$and a linear operator $S_{i} \in \mathcal{I}_{i}\left(E_{i}, G_{i}\right)$ such that for any $\varepsilon_{i}>0$ and $x_{i} \in E_{i}(i=1, \ldots, n)$

$$
\left\|R_{i}\left(x_{i}\right)\right\| \leq N_{i}\left(\varepsilon_{i}^{\prime}\right)\left\|S_{i}\left(x_{i}\right)\right\|+\varepsilon_{i}^{\prime}\left\|x_{i}\right\|
$$

where $\varepsilon_{1}^{\prime}=\frac{\varepsilon_{1}}{\|S\|}$ and $\varepsilon_{i}^{\prime}:=\varepsilon_{i}$ if $i=2, \ldots, n$. Hence, $\left\|T\left(x_{1}, \ldots, x_{n}\right)\right\|_{F} \leq$ $\|S\|\left\|R_{1}\left(x_{1}\right)\right\| \cdots\left\|R_{n}\left(x_{n}\right)\right\|$

$\leq\|S\|\left(N_{1}\left(\varepsilon_{1}^{\prime}\right)\left\|S_{1}\left(x_{1}\right)\right\|+\varepsilon_{1}^{\prime}\left\|x_{1}\right\|\right) \cdots\left(N_{n}\left(\varepsilon_{n}\right)\left\|S_{n}\left(x_{n}\right)\right\|+\varepsilon_{n}\left\|x_{n}\right\|\right)$.

The functions $\|S\| N_{1}\left(\varepsilon_{1} /\|S\|\right), N_{2}\left(\varepsilon_{2}\right), \ldots, N_{n}\left(\varepsilon_{n}\right)$ and $S_{1}, \ldots, S_{n}$ are what we were looking for.

Let us proceed with the converse. Suppose that for every $i=1, \ldots, n$ there exist a function $N_{i}: R^{+} \rightarrow R^{+}$and an operator $S_{i} \in \mathcal{I}_{i}\left(E_{i} ; G_{i}\right)$ such that $\left\|T\left(x_{1}, \ldots, x_{n}\right)\right\| \leq\left(N_{1}\left(\varepsilon_{1}\right)\left\|S_{1}\left(x_{1}\right)\right\|+\varepsilon_{1}\left\|x_{1}\right\|\right) \cdots\left(N_{n}\left(\varepsilon_{n}\right)\left\|S_{n}\left(x_{n}\right)\right\|+\varepsilon_{n}\left\|x_{n}\right\|\right)$, for all $x_{1} \in E_{1}, \ldots, x_{n} \in E_{n}$ and all $\varepsilon_{1}>0, \ldots, \varepsilon_{n}>0$. We will prove that $T_{i} \in \overline{\mathcal{I}}_{i}^{i n j}\left(E_{i} ; \mathcal{L}\left(E_{1},{ }^{[i]} ., E_{n} ; F\right)\right)$, for $i=1, \ldots, n$. It is enough to see it for a fixed $i$, since the argument is the same for the rest. Take for instance $i=1$. Let $\varepsilon>0$ and $x_{1} \in E_{1}$. Given arbitrary $x_{2} \in B_{E_{2}}, \ldots, x_{n} \in B_{E_{n}}$, choosing in (2) any $\varepsilon_{j}>$ $0(j=2, \ldots, n)$ and $\varepsilon_{1}=\varepsilon / K$, with $K=\left(N_{2}\left(\varepsilon_{2}\right)\left\|S_{2}\right\|+\varepsilon_{2}\right) \cdots\left(N_{n}\left(\varepsilon_{n}\right)\left\|S_{n}\right\|+\right.$ $\left.\varepsilon_{n}\right)$, it follows that $\left\|T_{1}\left(x_{1}\right)\left(x_{2}, \ldots, x_{n}\right)\right\|=\left\|T\left(x_{1}, \ldots, x_{n}\right)\right\|$

$\leq\left(N_{1}\left(\varepsilon_{1}\right)\left\|S_{1}\left(x_{1}\right)\right\|+\varepsilon_{1}\left\|x_{1}\right\|\right) \cdots\left(N_{n}\left(\varepsilon_{n}\right)\left\|S_{n}\left(x_{n}\right)\right\|+\varepsilon_{n}\left\|x_{n}\right\|\right)$

$\leq K\left(N_{1}\left(\varepsilon_{1}\right)\left\|S_{1}\left(x_{1}\right)\right\|+\varepsilon_{1}\left\|x_{1}\right\|\right)=K N_{1}\left(\varepsilon_{1}\right)\left\|S_{1}\left(x_{1}\right)\right\|+\varepsilon\left\|x_{1}\right\|$. Hence, denoting $\widehat{N}_{1}(\varepsilon)=K N_{1}(\varepsilon / K)$, we conclude that

$$
\left\|T_{1}\left(x_{1}\right)\right\|_{\mathcal{L}\left(E_{2}, \ldots, E_{n} ; F\right)} \leq \widehat{N}_{1}(\varepsilon)\left\|S_{1}\left(x_{1}\right)\right\|+\varepsilon\left\|x_{1}\right\|, \text { forevery }
$$

$\varepsilon>0$ and each $x_{1} \in E_{1}$.

\section{Thus}

$\mathrm{T}_{1} \in \overline{\mathcal{I}}^{i n j}\left(E_{1} ; \mathcal{L}\left(E_{2}, \ldots, E_{n} ; F\right)\right)$, and the proof is complete.

If $T$ satisfies the domination inequality (2) of Theorem 3 , then $T \in \mathcal{L}\left({\overline{\mathcal{I}_{1}}}^{i n j}, \ldots, \overline{\mathcal{I}}_{n}{ }^{i n j}\right)$. We will just give a sketch of this. For each $i=1, \ldots, n$, consider the positively homogeneous function

$$
\Phi_{i}(x):=\inf _{\varepsilon>0}\left\{N_{i}(\varepsilon)\left\|S_{i}(x)\right\|+\varepsilon\|x\|\right\}, \quad x \in E_{i},
$$

and its convexification

$$
\left\|x_{i}\right\|_{N_{i}, S_{i}}:=\inf \left\{\sum_{j=1}^{m} \Phi_{i}\left(x_{i j}\right): \sum_{j=1}^{m} x_{i j}=x_{i}\right\}, \quad x_{i} \in E_{i} .
$$


Let $E_{N_{i}, S_{i}}$ be the Banach space defined as the completion of the quotient space formed by the equivalence classes $x \equiv y \leftrightarrow\|x-y\|_{N_{i}, S_{i}}=0$. Note that the quotient map $j_{i}: E_{i} \rightarrow E_{N_{i}, S_{i}}$ is continuous and so

$$
\left\|x_{i}\right\|_{N_{i}, S_{i}} \leq K_{i} \Phi_{i}\left(x_{i}\right) \leq K_{i} N_{i}\left(\frac{\varepsilon_{i}}{K_{i}}\right)\left\|S_{i}\left(x_{i}\right)\right\|+\varepsilon_{i}\left\|x_{i}\right\|,
$$

for some constant $K_{i}>0$ and all $\varepsilon_{i}>0$ and all $x_{i} \in E_{i}$. Using (1), $j_{i} \in$ $\overline{\mathcal{I}}_{i}^{i n j}\left(E_{i} ; E_{N_{i}, S_{i}}\right), i=1, \ldots, n$. Moreover, $T$ admits the following factorization through the product of the Banach spaces $E_{N_{i}, S_{i}}$

$$
\begin{aligned}
& E_{1} \times \cdots \times E_{n} \longrightarrow F \\
& \left(j_{1}, \ldots, j_{n}\right) \cdots \cdots, \quad \rightarrow \\
& E_{N_{1}, S_{1}} \times \cdots \times E_{N_{n}, S_{n}}
\end{aligned}
$$

where $S$ is a continuous multilinear map.

Our aim now is to introduce a multilinear measure that characterizes the operators that belong to $\overline{\mathcal{M}}_{n}^{i n j}$, for a given $n$-ideal $\mathcal{M}_{n}$. It turns out that this measure will coincide with the inner measure of the linearization of the multilinear mapping for certain class of multi-ideals. The following result is a preliminary step before our objective.

Let $\mathcal{M}_{n}$ be an ideal of $n$-linear operators and $T \in \mathcal{L}\left(E_{1}, \ldots, E_{n} ; F\right)$. The following assertions are equivalent.

(a) $T \in \mathcal{M}_{n}^{\text {inj }}\left(E_{1}, \ldots, E_{n} ; F\right)$.

(b) There are a Banach space $G$ and an operator $R \in \mathcal{M}_{n}\left(E_{1}, \ldots, E_{n} ; G\right)$ such that

$$
\left\|\sum_{j=1}^{m} T\left(x_{1}^{j}, \ldots, x_{n}^{j}\right)\right\| \leq\left\|\sum_{j=1}^{m} R\left(x_{1}^{j}, \ldots, x_{n}^{j}\right)\right\|,
$$

for all $m \in N$ and all $x_{1}^{j} \in E_{1}, \ldots, x_{n}^{j} \in E_{n}, j=1, \ldots, m$.

(a) $\Longrightarrow$ (b) It is enough to take $R:=J_{F} \circ T$.

(b) $\Longrightarrow$ (a) Consider the normed space $G_{0}$ defined as the linear span of $R\left(E_{1} \times\right.$ $\left.\cdots \times E_{n}\right) \subseteq G$, i.e. the normed space of all vectors of the form $\sum_{j=1}^{m} R\left(x_{1}^{j}, \ldots, x_{n}^{j}\right) \in$ $G$, with $m \in N$ and $x_{1}^{j} \in E_{1}, \ldots, x_{n}^{j} \in E_{n}, j=1, \ldots, m$. Now observe that the linear operator $S_{0}: G_{0} \rightarrow F$ given by

$$
S_{0}\left(\sum_{j=1}^{m} R\left(x_{1}^{j}, \ldots, x_{n}^{j}\right)\right):=\sum_{j=1}^{m} T\left(x_{1}^{j}, \ldots, x_{n}^{j}\right),
$$

is well-defined. This can be checked using the assumption in (b) and taking into account that, for each $j$, it holds that $\mathrm{T}\left(\mathrm{x}_{1}^{j}, \ldots, x_{n}^{j}\right)-T\left(y_{1}^{j}, \ldots, y_{n}^{j}\right)=$ $T\left(x_{1}^{j}-y_{1}^{j}, x_{2}^{j}, \ldots, x_{n}^{j}\right)+T\left(y_{1}^{j}, x_{2}^{j}-y_{2}^{j}, x_{3}^{j}, \ldots, x_{n}^{j}\right)+$ $+T\left(y_{1}^{j}, y_{2}^{j}, x_{3}^{j}-y_{3}^{j}, x_{4}^{j}, \ldots, x_{n}^{j}\right)+\cdots+T\left(y_{1}^{j}, y_{2}^{j}, \ldots, y_{n-1}^{j}, x_{n}^{j}-y_{n}^{j}\right)$. We can extend $S_{0}$ to the completion $\bar{G}_{0}$ of $G_{0}$ : write $S$ for this extension. Since $\ell^{\infty}\left(B_{F^{*}}\right)$ has 
the metric extension property, we obtain a new operator $S^{e x t}: G \rightarrow \ell^{\infty}\left(B_{F^{*}}\right)$ which satisfies that $J_{F} \circ T=S^{e x t} \circ R$. Due to $R \in \mathcal{M}_{n}\left(E_{1}, \ldots, E_{n} ; G\right)$, we get that $J_{F} \circ T \in \mathcal{M}_{n}\left(E_{1}, \ldots, E_{n} ; \ell^{\infty}\left(B_{F^{*}}\right)\right)$.

In 2010 Botelho, Galindo and Pellegrini [8, Theorem 2.4] proved that, given $T \in \mathcal{L}\left(E_{1}, \ldots, E_{n} ; F\right), T \in \overline{\mathcal{M}}_{n}^{i n j}\left(E_{1}, \ldots, E_{n} ; F\right)$ if, and only if, for each $\varepsilon>0$ there is a Banach space $Z$ and $R \in \mathcal{M}_{n}\left(E_{1}, \ldots, E_{n} ; Z\right)$ such that

$$
\left\|\sum_{j=1}^{m} T\left(x_{1}^{j}, \ldots, x_{n}^{j}\right)\right\|_{F} \leq \varepsilon \sum_{j=1}^{m}\left\|x_{1}^{j}\right\|_{E_{1}} \cdots\left\|x_{n}^{j}\right\|_{E_{n}}+\left\|\sum_{j=1}^{m} R\left(x_{1}^{j}, \ldots, x_{n}^{j}\right)\right\|_{Z},
$$

for any $m \in N$ and $x_{1}^{j} \in E_{1}, \ldots, x_{n}^{j} \in E_{n}, j=1, \ldots, m$.

Inspired by (3), we next introduce a measure $\widetilde{\beta}_{\mathcal{M}_{n}}$ which satisfies that $\widetilde{\beta}_{\mathcal{M}_{n}}(T)=0$ if and only if $T \in \overline{\mathcal{M}}_{n}{ }^{i n j}$. Thus, in particular, $\widetilde{\beta}_{\mathcal{M}_{n}}(T)=0$ if and only if $T \in \mathcal{M}_{n}$, whenever $\mathcal{M}_{n}$ is a closed injective ideal of $n$-linear operators.

Let $\mathcal{M}_{n}$ be an ideal of $n$-linear operators. For $T \in \mathcal{L}\left(E_{1}, \ldots, E_{n} ; F\right)$, $\widetilde{\beta}_{\mathcal{M}_{n}}(T)=\widetilde{\beta}_{\mathcal{M}_{n}}\left(T: E_{1} \times \cdots \times E_{n} \rightarrow F\right):=$ $\inf \left\{\varepsilon>0:\right.$ thereisaBanachspaceZandR $\in \mathcal{M}_{n}\left(E_{1}, \ldots, E_{n} ; Z\right)$ suchthat $\left\|\sum_{j=1}^{m} T\left(x_{1}^{j}, \ldots, x_{n}^{j}\right)\right\|_{F} \leq \varepsilon \sum_{j=1}^{m}\left\|x_{1}^{j}\right\| \cdots\left\|x_{n}^{j}\right\|+\left\|\sum_{j=1}^{m} R\left(x_{1}^{j}, \ldots, x_{n}^{j}\right)\right\|$, for all $m \in N$ and all $\left.x_{1}^{j} \in E_{1}, \ldots, x_{n}^{j} \in E_{n}, j=1, \ldots, m\right\}$.

It is very easy to check that if $\mathcal{M}_{n}=\left[\mathcal{I}_{1}, \ldots, \mathcal{I}_{n}\right]$, where $\mathcal{I}_{1}, \ldots, \mathcal{I}_{n}$ are linear operator ideals, it holds that

$$
\beta_{\left[\mathcal{I}_{1}, \ldots, \mathcal{I}_{n}\right]}(T) \leq \widetilde{\beta}_{\left[\mathcal{I}_{1}, \ldots, \mathcal{I}_{n}\right]}(T), \text { forevery } T \in \mathcal{L}\left(E_{1}, \ldots, E_{n} ; F\right) .
$$

Nevertheless, $\beta_{\left[\mathcal{I}_{1}, \ldots, \mathcal{I}_{n}\right]}$ and $\widetilde{\beta}_{\left[\mathcal{I}_{1}, \ldots, \mathcal{I}_{n}\right]}$ do not coincide in general. In fact, if $\varepsilon>$ $\widetilde{\beta}_{\left[\mathcal{I}_{1}, \ldots, \mathcal{I}_{n}\right]}(T)$, there are a Banach space $Z$ and an operator $R \in\left[\mathcal{I}_{1}, \ldots, \mathcal{I}_{n}\right]\left(E_{1}, \ldots, E_{n} ; Z\right)$ such that, for all $x_{1} \in E_{1}, \ldots, x_{n} \in E_{n},\left\|T\left(x_{1}, \ldots, x_{n}\right)\right\|_{F} \leq \varepsilon\left\|x_{1}\right\| \cdots\left\|x_{n}\right\|+$ $\left\|R\left(x_{1}, \ldots, x_{n}\right)\right\|$ $\leq \varepsilon\left\|x_{1}\right\| \cdots\left\|x_{n}\right\|+\left\|R_{i}\left(x_{i}\right)\right\|\left\|x_{1}\right\| .[i] .\left\|x_{n}\right\|$ whatever $i=1, \ldots, n$. Therefore,

$$
\left\|T\left(x_{1}, \ldots, x_{n}\right)\right\|_{F} \leq \varepsilon\left\|x_{1}\right\| \cdots\left\|x_{n}\right\|+\min _{i \in\{1, \ldots, n\}}\left\{\left\|R_{i}\left(x_{i}\right)\right\|\left\|x_{1}\right\| \stackrel{[i]}{\cdot}\left\|x_{n}\right\|\right\} .
$$

This implies $\beta_{\left[\mathcal{I}_{1}, \ldots, \mathcal{I}_{n}\right]}(T) \leq \widetilde{\beta}_{\left[\mathcal{I}_{1}, \ldots, \mathcal{I}_{n}\right]}(T)$. However, choosing $\mathcal{I}_{1}=\mathcal{I}_{2}=\mathcal{A}$, the ideal of approximable operators, and taking $A$ the bilinear form considered in Remark 3, we know that $\beta_{[\mathcal{A}, \mathcal{A}]}(A)=0$ because $A \in\left[\overline{\mathcal{A}}^{i n j}, \overline{\mathcal{A}}^{i n j}\right](E, E ; F)$, but $\widetilde{\beta}_{[\mathcal{A}, \mathcal{A}]}(A)>0$ since $A \notin \overline{[\mathcal{A}, \mathcal{A}]}^{i n j}(E, E ; F)$.

We note that both measures we have introduced so far, $\beta_{\left[\mathcal{I}_{1}, \ldots, \mathcal{I}_{n}\right]}$ and $\widetilde{\beta}_{\mathcal{M}_{n}}$, coincide with the measure $\beta_{\mathcal{I}}$ when $n=1$ and $\mathcal{I}$ is any ideal of linear operators. For instance, if $\varepsilon>\beta_{\mathcal{I}}(T)$ there exist a Banach space $Z$ and an operator $R \in$ $\mathcal{I}(E ; Z)$ such that

$$
\|T(x)\|_{F} \leq \varepsilon\|x\|_{E}+\|R(x)\|_{Z}, \text { forall } x \in E .
$$


Thus, for any $m \in N$ and $x^{j} \in E, j=1, \ldots m$,

$$
\left\|\sum_{j=1}^{m} T\left(x^{j}\right)\right\|_{F}=\left\|T\left(\sum_{j=1}^{m} x^{j}\right)\right\|_{F} \leq \varepsilon \sum_{j=1}^{m}\left\|x^{j}\right\|_{E}+\left\|\sum_{j=1}^{m} R\left(x^{j}\right)\right\|_{Z},
$$

and so $\varepsilon>\widetilde{\beta}_{\mathcal{I}}(T)$. The other inequality $\beta_{\mathcal{I}}(T) \leq \widetilde{\beta}_{\mathcal{I}}(T)$ is also trivial.

Any $R \in \mathcal{L}\left(\mathcal{I}_{1}, \ldots, \mathcal{I}_{n}\right)\left(E_{1}, \ldots, E_{n} ; F\right)$ factors as $R=B \circ\left(S_{1}, \ldots, S_{n}\right)$, for some $S_{i} \in \mathcal{I}_{i}\left(E_{i} ; G_{i}\right)$, some $B \in \mathcal{L}\left(G_{1}, \ldots, G_{n} ; F\right)$ and some Banach space $G_{i}$, $i=1, \ldots, n$. Then

$$
\left\|R\left(x_{1}, \ldots, x_{n}\right)\right\| \leq\|B\|\left\|S_{1}\left(x_{1}\right)\right\| \cdots\left\|S_{n}\left(x_{n}\right)\right\| .
$$

Hence, it is easy to conclude that $\beta_{\left[\mathcal{I}_{1}, \ldots, \mathcal{I}_{n}\right]} \leq \widetilde{\beta}_{\mathcal{L}\left(\mathcal{I}_{1}, \ldots, \mathcal{I}_{n}\right)}$.

Let $\mathcal{M}_{n}$ be an ideal of $n$-linear operators. For $T \in \mathcal{L}\left(E_{1}, \ldots, E_{n} ; F\right)$, $\widetilde{\beta}_{\mathcal{M}_{n}}(T)=\inf \left\{\varepsilon>0:\right.$ thereareaBanachspaceZandR $\in \mathcal{M}_{n}\left(E_{1}, \ldots, E_{n} ; Z\right)$ sothat $\left\|\sum_{j=1}^{m} T\left(x_{1}^{j}, \ldots, x_{n}^{j}\right)\right\|_{F} \leq \varepsilon \cdot \pi\left(\sum_{j=1}^{m} x_{1}^{j} \otimes \cdots \otimes x_{n}^{j}\right)+\left\|\sum_{j=1}^{m} R\left(x_{1}^{j}, \ldots, x_{n}^{j}\right)\right\|$, for any $m \in N$ and all $\left.x_{1}^{j} \in E_{1}, \ldots, x_{n}^{j} \in E_{n}, j=1, \ldots, m\right\}$.

Clearly, $\widetilde{\beta}_{\mathcal{M}_{n}}$ is less than or equal to the above infimum. To show the converse inequality take $T \in \mathcal{L}\left(E_{1}, \ldots, E_{n} ; F\right)$. Let $\varepsilon>\widetilde{\beta}_{\mathcal{M}_{n}}(T)$ and let $x_{1}^{j} \in$ $E_{1}, \ldots, x_{n}^{j} \in E_{n}, j=1, \ldots, m$. Consider the tensor $\theta=\sum_{j=1}^{m} x_{1}^{j} \otimes \cdots \otimes x_{n}^{j}$ and take $\delta>0$. We can find a representation of $\theta=\sum_{j=1}^{l} y_{1}^{j} \otimes \cdots \otimes y_{n}^{j}$ such that

$$
\sum_{j=1}^{l}\left\|y_{1}^{j}\right\| \cdots\left\|y_{n}^{j}\right\| \leq \pi(\theta)+\delta .
$$

Then, for some Banach space $Z$ and some operator $R \in \mathcal{M}_{n}\left(E_{1}, \ldots, E_{n} ; Z\right)$, we have (adding zeros if necessary) $\left\|\sum_{j=1}^{m} T\left(x_{1}^{j}, \ldots, x_{n}^{j}\right)\right\|=\| T_{L}\left(\sum_{j=1}^{m} x_{1}^{j} \otimes \cdots \otimes\right.$ $\left.x_{n}^{j}\right)\|=\| T_{L}\left(\sum_{j=1}^{l} y_{1}^{j} \otimes \cdots \otimes y_{n}^{j}\right) \|$ $=\left\|\sum_{j=1}^{l} T\left(y_{1}^{j}, \ldots, y_{n}^{j}\right)\right\| \leq \varepsilon \sum_{j=1}^{l}\left\|y_{1}^{j}\right\| \cdots\left\|y_{n}^{j}\right\|+\left\|\sum_{j=1}^{l} R\left(y_{1}^{j}, \ldots, y_{n}^{j}\right)\right\|$ $\leq \varepsilon(\pi(\theta)+\delta)+\left\|\sum_{j=1}^{m} R\left(x_{1}^{j}, \ldots, x_{n}^{j}\right)\right\|$. As $\delta$ is arbitrary, the conclusion follows.

As mentioned before, in $\left[8\right.$, Theorem 2.4] it is proved that given $T \in \mathcal{L}\left(E_{1}, \ldots, E_{n} ; F\right)$, then $\widetilde{\beta}_{\mathcal{M}_{n}}(T)=0$ if, and only if, $T \in \overline{\mathcal{M}}_{n}^{i n j}\left(E_{1}, \ldots, E_{n} ; F\right)$. Proposition 3 seems to indicate that, given a multi-ideal $\mathcal{M}, \widetilde{\beta}_{\mathcal{M}_{n}}(T)$ is close to $\beta_{\mathcal{M}_{1}}\left(T_{L}\right)$, and one could even think that both values coincide. This will be the case if $\mathcal{M}$ is closed under linearization, i.e. if $T_{L} \in \mathcal{M}_{1}$ whenever $T \in \mathcal{M}$. However, it cannot be ensured in general that $T_{L} \in \mathcal{M}_{1}$ when $T \in \mathcal{M}$. Indeed, Botelho [7] gave an example of a $p$-dominated $n$-homogeneous polynomial $P_{0}$ which is not weakly compact. Therefore, its linearization $L_{0}$ is not weakly compact. Given $T \in \mathcal{L}\left({ }^{n} E ; F\right)$ we denote $\widehat{T}$ the polynomial $\widehat{T}(x)=T(x, \ldots, x)$. Consider the ideal $\mathcal{M}_{0}$ of all continuous $n$-linear operators $T$ such that $\widehat{T}$ is 
$p$-dominated. Then, the unique symmetric $n$-linear operator $T_{0}$ associated to $P_{0}$ satisfies that $T_{0} \in \mathcal{M}_{0}$, but its linearization $L_{0}$ is not absolutely $p$-summing, that is, $L_{0} \notin\left(\mathcal{M}_{0}\right)_{1}$.

Let us show a class of multi-ideals $\mathcal{M}$ for which $T_{L} \in \mathcal{M}_{1}$ if and only if $T \in \mathcal{M}$. Let $\mathcal{I}$ be a linear operator ideal. The composition multi-ideal $\mathcal{I} \circ \mathcal{L}$ is formed by all compositions of continuous multilinear mappings with elements of $\mathcal{I}$; that is, an $n$-linear operator $T$ belongs to the component $\mathcal{I} \circ \mathcal{L}\left(E_{1}, \ldots, E_{n} ; F\right)$ if there are a Banach space $G$, an $n$-linear operator $S \in \mathcal{L}\left(E_{1}, \ldots, E_{n} ; G\right)$ and a linear operator $R \in \mathcal{I}(G ; F)$ such that $T=R \circ S$. By [9, Proposition 3.2] an $n$-linear operator $T \in \mathcal{L}\left(E_{1}, \ldots, E_{n} ; F\right)$ belongs to $\mathcal{I} \circ \mathcal{L}$ if and only if its linearization $T_{L}$ belongs to $\mathcal{I}\left(E_{1} \hat{\otimes}_{\pi} \cdots \hat{\otimes}_{\pi} E_{n} ; F\right)$. Therefore,

$$
\widetilde{\beta}_{\mathcal{I} \circ \mathcal{L}}(T)=\beta_{\mathcal{I}}\left(T_{L}\right), \text { forany } T \in \mathcal{L}\left(E_{1}, \ldots, E_{n} ; F\right) .
$$

Examples of this kind of composition multi-ideals are the compact multilinear operators, the weakly compact multilinear operators (both as consequences of the work of Pełczyński [27]), that are composition of continuous multilinear operators with compact operators and weakly compact operators respectively, and the factorable strongly $p$-summing multilinear operators introduced in [28], that can be seen as composition of continuous multilinear operators with absolutely $p$-summing linear operators (see also [32]). Other examples can be found in [9].

Let $\mathcal{I}$ be a linear operator ideal and let $T \in \mathcal{L}\left(E_{1}, \ldots, E_{n} ; F\right)$. The following statements are equivalent:

(a) $T \in \overline{\mathcal{I} \circ \mathcal{L}^{i n j}}\left(E_{1}, \ldots, E_{n} ; F\right)$.

(b) $T_{L} \in \overline{\mathcal{I}}^{i n j}\left(E_{1} \hat{\otimes}_{\pi} \cdots \hat{\otimes}_{\pi} E_{n} ; F\right)$.

(c) $\widetilde{\beta}_{\mathcal{I} \circ \mathcal{L}}(T)=0$.

(d) $\beta_{\mathcal{I}}\left(T_{L}\right)=0$.

Let $\mathcal{I}$ be a linear operator ideal. Then, $\overline{\mathcal{I} \circ \mathcal{L}^{i n j}}=\overline{\mathcal{I}}^{i n j} \circ \mathcal{L}$.

This last Corollary has been already stated in [8, Proposition 4.6].

\section{Interpolation properties of the measures}

Before of establishing the results of this section, we recall some basic definitions about interpolation theory.

It is said to be that $\bar{A}=\left(A_{0}, A_{1}\right)$ is a Banach couple if $A_{0}$ and $A_{1}$ are Banach spaces which are continuously embedded in some Hausdorff topological vector space. The spaces $\Sigma(\bar{A}):=A_{0}+A_{1}$ and $\Delta(\bar{A}):=A_{0} \cap A_{1}$ become Banach spaces when endowed with the norms $K(1, \cdot)$ and $J(1, \cdot)$, respectively, where the $K$ and $J$ functionals are defined, for $t>0$, by

$K(t, a)=K(t, a ; \bar{A}):=\inf \left\{\left\|a_{0}\right\|_{A_{0}}+t\left\|a_{1}\right\|_{A_{1}}: a=a_{0}+a_{1}, a_{i} \in A_{i}\right\}, a \in \Sigma(\bar{A})$. 


$$
J(t, a)=J(t, a ; \bar{A}):=\max \left\{\|a\|_{A_{0}}, t\|a\|_{A_{1}}\right\}, a \in \Delta(\bar{A}) .
$$

A Banach space $A$ is called an intermediate space with respect to $\bar{A}=$

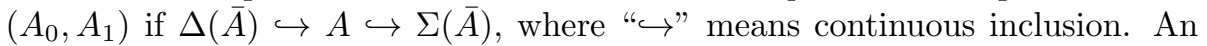
intermediate space $A$ with respect to $\bar{A}=\left(A_{0}, A_{1}\right)$ is said to be of class $C_{J}(\theta ; \bar{A})$, where $0<\theta<1$, if there exists a constant $C>0$ such that for all $t>0$ and $a \in A_{0} \cap A_{1}$,

$$
\|a\|_{A} \leq C t^{-\theta} J(t, a) .
$$

The real interpolation space $\left(A_{0}, A_{1}\right)_{\theta, q}$ and the complex interpolation space $\left(A_{0}, A_{1}\right)_{[\theta]}$ are important examples of spaces of class $C_{J}(\theta ; \bar{A})$. We refer to the books [5] and [33] for wide information about fundamentals of interpolation theory.

Next we investigate the behavior under interpolation of the measures $\beta_{\left[\mathcal{I}_{1}, \ldots, \mathcal{I}_{n}\right]}$ and $\widetilde{\beta}_{\mathcal{M}_{n}}$, introduced in Section 3. Our techniques are inspired by ideas used in [11, Theorem 3.3] and [12, Theorem 3.1] (see also [13]) for the linear case.

Let $\mathcal{M}_{n}$ be any ideal of $n$-linear operators, let $E_{1}, \ldots, E_{n}$ be Banach spaces and let $\bar{F}=\left(F_{0}, F_{1}\right)$ be a Banach couple. Assume that $F$ is of class $\mathcal{C}_{J}(\theta, \bar{F})$ with constant $C$. For $T \in \mathcal{L}\left(E_{1}, \ldots, E_{n} ; \Delta(\bar{F})\right), \widetilde{\beta}_{\mathcal{M}_{n}}\left(T: E_{1} \times \cdots \times E_{n} \rightarrow F\right) \leq$ $C \widetilde{\beta}_{\mathcal{M}_{n}}\left(T: E_{1} \times \cdots \times E_{n} \rightarrow F_{0}\right)^{1-\theta} \widetilde{\beta}_{\mathcal{M}_{n}}\left(T: E_{1} \times \cdots \times E_{n} \rightarrow F_{1}\right)^{\theta}$.

Let $\varepsilon_{k}>\widetilde{\beta}_{\mathcal{M}_{n}}\left(T: E_{1} \times \cdots \times E_{n} \rightarrow F_{k}\right), k=0,1$. Then, for certain Banach space $Z_{k}$ and $n$-linear operator $R_{k} \in \mathcal{M}_{n}\left(E_{1}, \ldots, E_{n} ; Z_{k}\right)$, it holds that

$$
\left\|\sum_{j=1}^{m} T\left(x_{1}^{j}, \ldots, x_{n}^{j}\right)\right\|_{F_{k}} \leq \varepsilon_{k} \sum_{j=1}^{m}\left\|x_{1}^{j}\right\| \ldots\left\|x_{n}^{j}\right\|+\left\|\sum_{j=1}^{m} R_{k}\left(x_{1}^{j}, \ldots, x_{n}^{j}\right)\right\|_{Z_{k}} \quad(k=0,1),
$$

for all $m \in N$ and $x_{1}^{j} \in E_{1}, \ldots, x_{n}^{j} \in E_{n}, j=1, \ldots, m$.

Let $t>0$. We denote $Z:=\left(Z_{0} \oplus Z_{1}\right)_{1}$ and $\varepsilon:=\min \left\{\varepsilon_{0}, \varepsilon_{1}\right\}$. Moreover, we define the $n$-linear operator $R \in \mathcal{L}\left(E_{1}, \ldots, E_{n} ; Z\right)$ by

$$
R\left(x_{1}, \ldots, x_{n}\right):=\frac{C t^{-\theta} \max \left\{\varepsilon_{0}, t \varepsilon_{1}\right\}}{\varepsilon}\left(R_{0}\left(x_{1}, \ldots, x_{n}\right), R_{1}\left(x_{1}, \ldots, x_{n}\right)\right) .
$$

Since $R=\frac{C t^{-\theta} \max \left\{\varepsilon_{0}, t \varepsilon_{1}\right\}}{\varepsilon}\left(\psi_{0} \circ R_{0}+\psi_{1} \circ R_{1}\right)$, where $\psi_{k}: Z_{k} \rightarrow Z$ is the natural inclusion $(k=0,1)$, then $R \in \mathcal{M}_{n}\left(E_{1}, \ldots, E_{n} ; Z\right)$.

For any $m \in N$ and $x_{1}^{j} \in E_{1}, \ldots, x_{n}^{j} \in E_{n}, j=1, \ldots, m$, it follows from (4) that $\left\|\sum_{j=1}^{m} T\left(x_{1}^{j}, \cdots, x_{n}^{j}\right)\right\|_{F} \leq C t^{-\theta} J\left(t, \sum_{j=1}^{m} T\left(x_{1}^{j}, \ldots, x_{n}^{j}\right)\right)$

$\leq C t^{-\theta} \max \left\{t^{k}\left(\varepsilon_{k} \sum_{j=1}^{m}\left\|x_{1}^{j}\right\| \cdots\left\|x_{n}^{j}\right\|+\left\|\sum_{j=1}^{m} R_{k}\left(x_{1}^{j}, \ldots, x_{n}^{j}\right)\right\|_{Z_{k}}\right): k=0,1\right\}$ $\leq C t^{-\theta} \max \left\{t^{k} \varepsilon_{k}\left(\sum_{j=1}^{m}\left\|x_{1}^{j}\right\| \cdots\left\|x_{n}^{j}\right\|+\frac{1}{\varepsilon}\left\|\sum_{j=1}^{m} R_{k}\left(x_{1}^{j}, \ldots, x_{n}^{j}\right)\right\|_{Z_{k}}\right): k=\right.$ $0,1\}$

$\leq C t^{-\theta} \max \left\{\varepsilon_{0}, t \varepsilon_{1}\right\} \max \left\{\sum_{j=1}^{m}\left\|x_{1}^{j}\right\| \cdots\left\|x_{n}^{j}\right\|+\frac{1}{\varepsilon}\left\|\sum_{j=1}^{m} R_{k}\left(x_{1}^{j}, \ldots, x_{n}^{j}\right)\right\|_{Z_{k}}:\right.$ $k=0,1\}$ 


$$
\begin{aligned}
& \leq C t^{-\theta} \max \left\{\varepsilon_{0}, t \varepsilon_{1}\right\}\left[\sum_{j=1}^{m}\left\|x_{1}^{j}\right\| \cdots\left\|x_{n}^{j}\right\|+\right. \\
& \left.\quad+\frac{1}{\varepsilon}\left(\left\|\sum_{j=1}^{m} R_{0}\left(x_{1}^{j}, \ldots, x_{n}^{j}\right)\right\|_{Z_{0}}+\left\|\sum_{j=1}^{m} R_{1}\left(x_{1}^{j}, \ldots, x_{n}^{j}\right)\right\|_{Z_{1}}\right)\right] \\
& =C t^{-\theta} \max \left\{\varepsilon_{0}, t \varepsilon_{1}\right\}\left[\sum_{j=1}^{m}\left\|x_{1}^{j}\right\| \cdots\left\|x_{n}^{j}\right\|+\frac{1}{\varepsilon} \frac{\varepsilon}{C t^{-\theta} \max \left\{\varepsilon_{0}, t \varepsilon_{1}\right\}}\left\|\sum_{j=1}^{m} R\left(x_{1}^{j}, \ldots, x_{n}^{j}\right)\right\|_{Z}\right] .
\end{aligned}
$$

Therefore, for any $t>0$, it holds that

$$
\widetilde{\beta}_{\mathcal{M}_{n}}\left(T: E_{1} \times \cdots \times E_{n} \rightarrow F\right) \leq C t^{-\theta} \max \left\{t^{k} \widetilde{\beta}_{\mathcal{M}_{n}}\left(T: E_{1} \times \cdots \times E_{n} \rightarrow F_{k}\right): k=0,1\right\} .
$$

We consider three possibilities:

i) If $\widetilde{\beta}_{\mathcal{M}_{n}}\left(T: E_{1} \times \cdots \times E_{n} \rightarrow F_{0}\right)=0$, then

$$
\widetilde{\beta}_{\mathcal{M}_{n}}\left(T: E_{1} \times \cdots \times E_{n} \rightarrow F\right) \leq C t^{1-\theta} \widetilde{\beta}_{\mathcal{M}_{n}}\left(T: E_{1} \times \cdots \times E_{n} \rightarrow F_{1}\right)
$$

for each $t>0$. Hence, $\widetilde{\beta}_{\mathcal{M}_{n}}\left(T: E_{1} \times \cdots \times E_{n} \rightarrow F\right)=0$.

ii) If $\widetilde{\beta}_{\mathcal{M}_{n}}\left(T: E_{1} \times \cdots \times E_{n} \rightarrow F_{1}\right)=0$, then

$$
\widetilde{\beta}_{\mathcal{M}_{n}}\left(T: E_{1} \times \cdots \times E_{n} \rightarrow F\right) \leq C t^{-\theta} \widetilde{\beta}_{\mathcal{M}_{n}}\left(T: E_{1} \times \cdots \times E_{n} \rightarrow F_{0}\right),
$$

for every $t>0$, and so $\widetilde{\beta}_{\mathcal{M}_{n}}\left(T: E_{1} \times \cdots \times E_{n} \rightarrow F\right)=0$.

iii) Assume that $\widetilde{\beta}_{\mathcal{M}_{n}}\left(T: E_{1} \times \cdots \times E_{n} \rightarrow F_{k}\right)>0$ for $k=0,1$. Then, for the particular choice $t:=\frac{\widetilde{\beta}_{\mathcal{M}_{n}}\left(T: E_{1} \times \cdots \times E_{n} \rightarrow F_{0}\right)}{\widetilde{\beta}_{\mathcal{M}_{n}}\left(T: E_{1} \times \cdots \times E_{n} \rightarrow F_{1}\right)}>0$ in (5), it turns out that $\widetilde{\beta}_{\mathcal{M}_{n}}\left(T: E_{1} \times \cdots \times E_{n} \rightarrow F\right) \leq$ $C \widetilde{\beta}_{\mathcal{M}_{n}}\left(T: E_{1} \times \cdots \times E_{n} \rightarrow F_{0}\right)^{1-\theta} \widetilde{\widetilde{\beta}}_{\mathcal{M}_{n}}\left(T: E_{1} \times \cdots \times E_{n} \rightarrow F_{1}\right)^{\theta}$.

Let $\mathcal{M}_{n}$ be a closed injective ideal of $n$-linear operators. Assume that $\bar{F}=$ $\left(F_{0}, F_{1}\right)$ is a Banach couple and $F$ is of class $\mathcal{C}_{J}(\theta, \bar{F})$. For $T \in \mathcal{L}\left(E_{1}, \ldots, E_{n} ; \Delta(\bar{F})\right)$, it follows that $T \in \mathcal{M}_{n}\left(E_{1}, \ldots, E_{n} ; F\right)$ whenever $T \in \mathcal{M}_{n}\left(E_{1}, \ldots, E_{n} ; F_{0}\right)$ or $T \in \mathcal{M}_{n}\left(E_{1}, \ldots, E_{n} ; F_{1}\right)$.

Theorem 4 recovers [11, Theorem 3.3] in the particular case $n=1$ and Corollary 4 can be read as a version of [19, Proposition 1.6] in the multilinear case.

We remark that even for $n=1$ a similar result to Theorem 4 does not hold in general if $T \in \mathcal{L}(\Sigma(\bar{E}) ; F)$, where $\bar{E}=\left(E_{0}, E_{1}\right)$ is a Banach couple and $F$ is a Banach space. To see it, we first recall that if $(\Omega, \Sigma)$ is a measurable space and $\mu$ is a $\sigma$-finite measure on $(\Omega, \Sigma)$, then it holds with equivalence of norms that (see [5, Theorem 5.3.1])

$$
\left(L_{\infty}, L_{1}\right)_{\theta, q}=L_{p, q}, \text { for } 0<\theta=1 / p<1,1 \leq q \leq \infty .
$$

As usual, the Lorentz space for the particular case $\Omega=[0,1]$ or $\Omega=[0, \infty)$, with the usual Lebesgue measure, will be denoted by $L_{p, q}[0,1]$ or $L_{p, q}[0, \infty)$, respectively.

Take $\mathcal{I}=\mathcal{S}$, the ideal of strictly singular operators, which is a closed injective operator ideal (see [22]). Let $\bar{E}=\left(L_{\infty}[0,1], L_{1}[0,1]\right)$, let $F=L_{1}[0,1]$ and let $T$ be the identity operator. Then $T: L_{\infty}[0,1] \rightarrow L_{1}[0,1]$ belongs to $\mathcal{I}$ (see 
[18]). However, by $(6)$, if $0<\theta=1 / p<1$, then $L_{p}[0,1]=\left(L_{\infty}[0,1], L_{1}[0,1]\right)_{\theta, p}$, but as it was pointed out by Beucher [6, Counterexample 2.4] the operator $T: L_{p}[0,1] \rightarrow L_{1}[0,1]$ does not belong to the ideal $\mathcal{I}$ since, according to Khintchine's inequality, the span of the Rademacher functions in $L_{p}[0,1]$ and $L_{1}[0,1]$ is isomorphic to $\ell_{2}$. Thus, the restriction of the identity operator $T$ to this subspace of $L_{p}[0,1]$ is an isomorphism into $L_{1}[0,1]$.

The following result provides an estimate in terms of the measures of the extreme restrictions $T: E_{1} \times \cdots \times E_{n} \rightarrow \Delta(\bar{F})$ and $T: E_{1} \times \cdots \times E_{n} \rightarrow \Sigma(\bar{F})$. It extends [13, Theorem 3.3]. Note that $\widetilde{\beta}_{\mathcal{M}_{n}}\left(T: E_{1} \times \cdots \times E_{n} \rightarrow \Delta(\bar{F})\right) \leq$ $\|T\|_{E_{1}, \ldots, E_{n}, \bar{F}}:=\max \left\{\|T\|_{\mathcal{L}\left(E_{1}, \ldots, E_{n} ; F_{k}\right)}: k=0,1\right\}$, and that our proof does not involve duality arguments.

Let $\mathcal{M}_{n}$ be any ideal of $n$-linear operators, let $E_{1}, \ldots, E_{n}$ be Banach spaces and let $\bar{F}=\left(F_{0}, F_{1}\right)$ be a Banach couple. Assume that $F$ is of class $\mathcal{C}_{J}(\theta, \bar{F})$ with constant $C$. For $T \in \mathcal{L}\left(E_{1}, \ldots, E_{n} ; \Delta(\bar{F})\right), \widetilde{\beta}_{\mathcal{M}_{n}}\left(T: E_{1} \times \cdots \times E_{n} \rightarrow F\right) \leq$ $4 C \widetilde{\beta}_{\mathcal{M}_{n}}\left(T: E_{1} \times \cdots \times E_{n} \rightarrow \Delta(\bar{F})\right)^{1-\Theta} \widetilde{\beta}_{\mathcal{M}_{n}}\left(T: E_{1} \times \cdots \times E_{n} \rightarrow \Sigma(\bar{F})\right)^{\Theta}$, where $\Theta=\min \{\theta, 1-\theta\}$.

Let $\eta>0$. We take any $t \geq 1$ such that

$$
t^{-\theta} \leq \eta \quad \text { and } \quad t^{\theta-1} \leq \eta
$$

Let $\sigma>\widetilde{\beta}_{\mathcal{M}_{n}}\left(T: E_{1} \times \cdots \times E_{n} \rightarrow \Sigma(\bar{F})\right)$. Then, it is possible to find a Banach space $H$ and an $n$-linear operator $R \in \mathcal{M}_{n}\left(E_{1}, \ldots, E_{n} ; H\right)$ such that

$$
\left\|\sum_{j=1}^{m} T\left(x_{1}^{j}, \ldots, x_{n}^{j}\right)\right\|_{\Sigma(\bar{F})} \leq \sigma \sum_{j=1}^{m}\left\|x_{1}^{j}\right\| \cdots\left\|x_{n}^{j}\right\|+\left\|\sum_{j=1}^{m} R\left(x_{1}^{j}, \ldots, x_{n}^{j}\right)\right\|_{H},
$$

for any $m \in N$ and $x_{1}^{j} \in E_{1}, \ldots, x_{n}^{j} \in E_{n}, j=1, \ldots, m$.

On the other hand, if $\delta>\widetilde{\beta}_{\mathcal{M}_{n}}\left(T: E_{1} \times \cdots \times E_{n} \rightarrow \Delta(\bar{F})\right)$ then, for certain Banach space $G$ and $n$-linear operator $S \in \mathcal{M}_{n}\left(E_{1}, \ldots, E_{n} ; G\right)$, it holds that

$$
\left\|\sum_{j=1}^{m} T\left(x_{1}^{j}, \ldots, x_{n}^{j}\right)\right\|_{\Delta(\bar{F})} \leq \delta \sum_{j=1}^{m}\left\|x_{1}^{j}\right\| \cdots\left\|x_{n}^{j}\right\|+\left\|\sum_{j=1}^{m} S\left(x_{1}^{j}, \ldots, x_{n}^{j}\right)\right\|_{G},
$$

for every $m \in N$ and $x_{1}^{j} \in E_{1}, \ldots, x_{n}^{j} \in E_{n}, j=1, \ldots, m$.

Let $\varepsilon>0$. We define $V:=(H \oplus G)_{1}$ and $P \in \mathcal{L}\left(E_{1}, \ldots, E_{n} ; V\right)$ given by

$$
P\left(x_{1}, \ldots, x_{n}\right):=(2+\varepsilon) t\left(R\left(x_{1}, \ldots, x_{n}\right), S\left(x_{1}, \ldots, x_{n}\right)\right) .
$$

Due to $P=(2+\varepsilon) t\left(\psi_{0} \circ R+\psi_{1} \circ S\right)$, where $\psi_{0}: H \rightarrow V$ and $\psi_{1}: G \rightarrow V$ are the natural inclusions, it follows that $P \in \mathcal{M}_{n}\left(E_{1}, \ldots, E_{n} ; V\right)$.

For any $m \in N$ and $x_{1}^{j} \in E_{1}, \ldots, x_{n}^{j} \in E_{n}, j=1, \ldots, m$, there exists a decomposition of $\sum_{j=1}^{m} T\left(x_{1}^{j}, \ldots, x_{n}^{j}\right)$ as $\sum_{j=1}^{m} T\left(x_{1}^{j}, \ldots, x_{n}^{j}\right)=y_{0}+y_{1}$, with $y_{k} \in F_{k}$ and

$$
\left\|y_{k}\right\|_{F_{k}} \leq\left\|y_{0}\right\|_{F_{0}}+\left\|y_{1}\right\|_{F_{1}} \leq(1+\varepsilon)\left\|\sum_{j=1}^{m} T\left(x_{1}^{j}, \ldots, x_{n}^{j}\right)\right\|_{\Sigma(\bar{F})}, \quad k=0,1 .
$$


It follows from (10) and (8) that

$$
\left\|y_{k}\right\|_{F_{k}} \leq(1+\varepsilon) \sigma \sum_{j=1}^{m}\left\|x_{1}^{j}\right\| \cdots\left\|x_{n}^{j}\right\|+(1+\varepsilon)\left\|\sum_{j=1}^{m} R\left(x_{1}^{j}, \ldots, x_{n}^{j}\right)\right\|_{H}, \quad k=0,1 .
$$

Since also $y_{k} \in \Delta(\bar{F}), k=0,1$, using (10) we obtain that $\left\|y_{k}\right\|_{F_{1-k}}=\| \sum_{j=1}^{m} T\left(x_{1}^{j}, \ldots, x_{n}^{j}\right)-$ $y_{1-k}\left\|_{F_{1-k}} \leq\right\| \sum_{j=1}^{m} T\left(x_{1}^{j}, \ldots, x_{n}^{j}\right)\left\|_{F_{1-k}}+\right\| y_{1-k} \|_{F_{1-k}}$ $\leq\left\|\sum_{j=1}^{m} T\left(x_{1}^{j}, \ldots, x_{n}^{j}\right)\right\|_{\Delta(\bar{F})}+(1+\varepsilon)\left\|\sum_{j=1}^{m} T\left(x_{1}^{j}, \ldots, x_{n}^{j}\right)\right\|_{\Sigma(\bar{F})}$ $\leq(2+\varepsilon)\left\|\sum_{j=1}^{m} T\left(x_{1}^{j}, \ldots, x_{n}^{j}\right)\right\|_{\Delta(\bar{F})}$, for $k=0,1$. By $(9)$,

$\left\|y_{k}\right\|_{F_{1-k}} \leq(2+\varepsilon) \delta \sum_{j=1}^{m}\left\|x_{1}^{j}\right\| \cdots\left\|x_{n}^{j}\right\|+(2+\varepsilon)\left\|\sum_{j=1}^{m} S\left(x_{1}^{j}, \ldots, x_{n}^{j}\right)\right\|_{G}, \quad k=0,1$.

Taking into account (4), (7), (11), (12) and the fact that $t \geq 1$, it holds that $\left\|\sum_{j=1}^{m} T\left(x_{1}^{j}, \ldots, x_{n}^{j}\right)\right\|_{F} \leq\left\|y_{0}\right\|_{F}+\left\|y_{1}\right\|_{F} \leq C t^{\theta} J\left(t^{-1}, y_{0}\right)+C t^{-\theta} J\left(t, y_{1}\right)$

$\leq C \eta t \max \left\{\left\|y_{0}\right\|_{F_{0}}, t^{-1}\left\|y_{0}\right\|_{F_{1}}\right\}+C \eta \max \left\{\left\|y_{1}\right\|_{F_{0}}, t\left\|y_{1}\right\|_{F_{1}}\right\}$

$\leq C \eta t \max \left\{(1+\varepsilon) \sigma \sum_{j=1}^{m}\left\|x_{1}^{j}\right\| \cdots\left\|x_{n}^{j}\right\|+(1+\varepsilon)\left\|\sum_{j=1}^{m} R\left(x_{1}^{j}, \ldots, x_{n}^{j}\right)\right\|_{H}\right.$, $\left.t^{-1}\left[(2+\varepsilon) \delta \sum_{j=1}^{m}\left\|x_{1}^{j}\right\| \cdots\left\|x_{n}^{j}\right\|+(2+\varepsilon)\left\|\sum_{j=1}^{m} S\left(x_{1}^{j}, \ldots, x_{n}^{j}\right)\right\|_{G}\right]\right\}$

$+C \eta \max \left\{(2+\varepsilon) \delta \sum_{j=1}^{m}\left\|x_{1}^{j}\right\| \cdots\left\|x_{n}^{j}\right\|+(2+\varepsilon)\left\|\sum_{j=1}^{m} S\left(x_{1}^{j}, \ldots, x_{n}^{j}\right)\right\|_{G}\right.$,

$\left.t\left[(1+\varepsilon) \sigma \sum_{j=1}^{m}\left\|x_{1}^{j}\right\| \cdots\left\|x_{n}^{j}\right\|+(1+\varepsilon)\left\|\sum_{j=1}^{m} R\left(x_{1}^{j}, \ldots, x_{n}^{j}\right)\right\|_{H}\right]\right\}$

$=2 C \eta \max \left\{(1+\varepsilon) \sigma t \sum_{j=1}^{m}\left\|x_{1}^{j}\right\| \cdots\left\|x_{n}^{j}\right\|+(1+\varepsilon) t\left\|\sum_{j=1}^{m} R\left(x_{1}^{j}, \ldots, x_{n}^{j}\right)\right\|_{H}\right.$,

$\left.(2+\varepsilon) \delta \sum_{j=1}^{m}\left\|x_{1}^{j}\right\| \cdots\left\|x_{n}^{j}\right\|+(2+\varepsilon)\left\|\sum_{j=1}^{m} S\left(x_{1}^{j}, \ldots, x_{n}^{j}\right)\right\|_{G}\right\}$

$\leq 2 C \eta \max \{(1+\varepsilon) \sigma t,(2+\varepsilon) \delta\} \sum_{j=1}^{m}\left\|x_{1}^{j}\right\| \cdots\left\|x_{n}^{j}\right\|+$

$+(1+\varepsilon) t\left\|\sum_{j=1}^{m} R\left(x_{1}^{j}, \ldots, x_{n}^{j}\right)\right\|_{H}+(2+\varepsilon)\left\|\sum_{j=1}^{m} S\left(x_{1}^{j}, \ldots, x_{n}^{j}\right)\right\|_{G}$

$\leq 2 C \eta \max \{(1+\varepsilon) \sigma t,(2+\varepsilon) \delta\} \sum_{j=1}^{m}\left\|x_{1}^{j}\right\| \cdots\left\|x_{n}^{j}\right\|+$

$+(2+\varepsilon) t\left(\left\|\sum_{j=1}^{m} R\left(x_{1}^{j}, \ldots, x_{n}^{j}\right)\right\|_{H}+\left\|\sum_{j=1}^{m} S\left(x_{1}^{j}, \ldots, x_{n}^{j}\right)\right\|_{G}\right)$

$=2 C \eta \max \{(1+\varepsilon) \sigma t,(2+\varepsilon) \delta\} \sum_{j=1}^{m}\left\|x_{1}^{j}\right\| \cdots\left\|x_{n}^{j}\right\|+\left\|\sum_{j=1}^{m} P\left(x_{1}^{j}, \ldots, x_{n}^{j}\right)\right\|_{V}$.

Hence,

$$
\widetilde{\beta}_{\mathcal{M}_{n}}\left(T: E_{1} \times \cdots \times E_{n} \rightarrow F\right) \leq 2 C \eta \max \{(1+\varepsilon) \sigma t,(2+\varepsilon) \delta\} .
$$

Therefore

$\widetilde{\beta}_{\mathcal{M}_{n}}\left(T: E_{1} \times \cdots \times E_{n} \rightarrow F\right) \leq 2 C \eta \cdot \quad \cdot \max \left\{\widetilde{\beta}_{\mathcal{M}_{n}}\left(T: E_{1} \times \cdots \times E_{n} \rightarrow \Sigma(\bar{F})\right) t, 2 \widetilde{\beta}_{\mathcal{M}_{n}}\left(T: E_{1} \times \cdots \times E_{n} \rightarrow \Delta(\bar{F})\right)\right\}$

We consider the following two cases: 
i) If $\widetilde{\beta}_{\mathcal{M}_{n}}\left(T: E_{1} \times \cdots \times E_{n} \rightarrow \Sigma(\bar{F})\right)=0$, then $\widetilde{\beta}_{\mathcal{M}_{n}}\left(T: E_{1} \times \cdots \times E_{n} \rightarrow F\right)=0$ as well, since $\eta$ is arbitrary.

ii) Assume that $\widetilde{\beta}_{\mathcal{M}_{n}}\left(T: E_{1} \times \cdots \times E_{n} \rightarrow \Sigma(\bar{F})\right)>0$. Note that $\widetilde{\beta}_{\mathcal{M}_{n}}(T:$ $\left.E_{1} \times \cdots \times E_{n} \rightarrow \Delta(\bar{F})\right)>0$ too, because $\widetilde{\beta}_{\mathcal{M}_{n}}\left(T: E_{1} \times \cdots \times E_{n} \rightarrow \Sigma(\bar{F})\right) \leq$ $\widetilde{\beta}_{\mathcal{M}_{n}}\left(T: E_{1} \times \cdots \times E_{n} \rightarrow \Delta(\bar{F})\right)$. Take

$\eta:=\max \left\{\left(\frac{\widetilde{\beta}_{\mathcal{M}_{n}}\left(T: E_{1} \times \cdots \times E_{n} \rightarrow \Sigma(\bar{F})\right)}{\widetilde{\beta}_{\mathcal{M}_{n}}\left(T: E_{1} \times \cdots \times E_{n} \rightarrow \Delta(\bar{F})\right)}\right)^{\theta},\left(\frac{\widetilde{\beta}_{\mathcal{M}_{n}}\left(T: E_{1} \times \cdots \times E_{n} \rightarrow \Sigma(\bar{F})\right)}{\widetilde{\beta}_{\mathcal{M}_{n}}\left(T: E_{1} \times \cdots \times E_{n} \rightarrow \Delta(\bar{F})\right)}\right)^{1-\theta}\right\}$.

The real number

$$
t:=\frac{\widetilde{\beta}_{\mathcal{M}_{n}}\left(T: E_{1} \times \cdots \times E_{n} \rightarrow \Delta(\bar{F})\right)}{\widetilde{\beta}_{\mathcal{M}_{n}}\left(T: E_{1} \times \cdots \times E_{n} \rightarrow \Sigma(\bar{F})\right)} \geq 1
$$

satisfies (7). If we denote $\Theta:=\min \{\theta, 1-\theta\}$ and substitute these concrete choices of $\eta$ and $t$ in (13), we obtain that $\widetilde{\beta}_{\mathcal{M}_{n}}\left(T: E_{1} \times \cdots \times E_{n} \rightarrow F\right) \leq$ $4 C \widetilde{\beta}_{\mathcal{M}_{n}}\left(T: E_{1} \times \cdots \times E_{n} \rightarrow \Delta(\bar{F})\right)$.

$\cdot \max \left\{\left(\frac{\widetilde{\beta}_{\mathcal{M}_{n}}\left(T: E_{1} \times \cdots \times E_{n} \rightarrow \Sigma(\bar{F})\right)}{\widetilde{\beta}_{\mathcal{M}_{n}}\left(T: E_{1} \times \cdots \times E_{n} \rightarrow \Delta(\bar{F})\right)}\right)^{\theta},\left(\frac{\widetilde{\beta}_{\mathcal{M}_{n}}\left(T: E_{1} \times \cdots \times E_{n} \rightarrow \Sigma(\bar{F})\right)}{\widetilde{\beta}_{\mathcal{M}_{n}}\left(T: E_{1} \times \cdots \times E_{n} \rightarrow \Delta(\bar{F})\right)}\right)^{1-\theta}\right\}$

$=4 C \widetilde{\beta}_{\mathcal{M}_{n}}\left(T: E_{1} \times \cdots \times E_{n} \rightarrow \Delta(\bar{F})\right)\left(\frac{\widetilde{\beta}_{\mathcal{M}_{n}}\left(T: E_{1} \times \cdots \times E_{n} \rightarrow \Sigma(\bar{F})\right)}{\widetilde{\beta}_{\mathcal{M}_{n}}\left(T: E_{1} \times \cdots \times E_{n} \rightarrow \Delta(\bar{F})\right)}\right)^{\Theta}$

$=4 C \widetilde{\beta}_{\mathcal{M}_{n}}\left(T: E_{1} \times \cdots \times E_{n} \rightarrow \Delta(\bar{F})\right)^{1-\Theta} \widetilde{\beta}_{\mathcal{M}_{n}}\left(T: E_{1} \times \cdots \times E_{n} \rightarrow \Sigma(\bar{F})\right)^{\Theta}$.

Let $\mathcal{M}_{n}$ be a closed injective ideal of $n$-linear operators. Assume that $\bar{F}=$ $\left(F_{0}, F_{1}\right)$ is a Banach couple and $F$ is of class $\mathcal{C}_{J}(\theta, \bar{F})$. For $T \in \mathcal{L}\left(E_{1}, \ldots, E_{n} ; \Delta(\bar{F})\right)$, it follows that $T \in \mathcal{M}_{n}\left(E_{1}, \ldots, E_{n} ; F\right)$ if and only if $T \in \mathcal{M}_{n}\left(E_{1}, \ldots, E_{n} ; \Sigma(\bar{F})\right)$.

We can use Corollaries 4 and 4 to establish results on the interpolation of certain classes of multilinear operators. Namely when $\mathcal{M}_{n}=\left[{\overline{\mathcal{I}_{1}}}^{i n j}, \ldots,{\overline{\mathcal{I}_{n}}}^{i n j}\right]$, where $\mathcal{I}_{1}, \ldots, \mathcal{I}_{n}$ are ideals of linear operators, it holds that $\mathcal{M}_{n}$ is a closed injective ideal of $n$-linear operators and so Corollaries 4 and 4 can be applied to $\mathcal{M}_{n}$. In particular, let us consider $\mathcal{I}$ to be any of the following ideals: (the closed injective ideal of) compact operators, weakly compact operators, strictly singular operators, Rosenthal operators, Banach-Saks operators, or decomposing operators (also called Asplund operators). Then, if $\mathcal{M}_{n}=[\mathcal{I}, \ldots, \mathcal{I}]$ we obtain an extension to the multilinear case of some interpolation results for these ideals of linear operators established in the literature (see for example [5, Theorem 3.8.1(ii)], [19, Proposition 1.6], [6, Proposition 2.1] and [25, Proposition 5]).

On the other hand, the previous interpolation formulas can be applied to provide, for instance, upper estimates for the measure $\widetilde{\beta}_{\mathcal{M}_{n}}\left(T: E_{1} \times \cdots \times E_{n} \rightarrow\right.$ $\left.L_{p, q}[0, \infty)\right)$ for any Lorentz space $L_{p, q}[0, \infty)$. Thus, because of $(6)$, the following logarithmically convex inequalities hold (for adequate $C>0$ in each case): $\widetilde{\beta}_{\mathcal{M}_{n}}\left(T: E_{1} \times \cdots \times E_{n} \rightarrow L_{p, q}[0, \infty)\right) \leq$

$C \widetilde{\beta}_{\mathcal{M}_{n}}\left(T: E_{1} \times \cdots \times E_{n} \rightarrow L_{\infty}[0, \infty)\right)^{1-\frac{1}{p}} \widetilde{\beta}_{\mathcal{M}_{n}}\left(T: E_{1} \times \cdots \times E_{n} \rightarrow L_{1}[0, \infty)\right)^{\frac{1}{p}}$ and $\widetilde{\beta}_{\mathcal{M}_{n}}\left(T: E_{1} \times \cdots \times E_{n} \rightarrow L_{p, q}[0, \infty)\right) \leq$ 
$C \widetilde{\beta}_{\mathcal{M}_{n}}\left(T: E_{1} \times \cdots \times E_{n} \rightarrow L_{1}[0, \infty) \cap L_{\infty}[0, \infty)\right)^{\max \left\{\frac{1}{p}, 1-\frac{1}{p}\right\}}$

. $\widetilde{\beta}_{\mathcal{M}_{n}}\left(T: E_{1} \times \cdots \times E_{n} \rightarrow L_{1}[0, \infty)+L_{\infty}[0, \infty)\right)^{\min \left\{\frac{1}{p}, 1-\frac{1}{p}\right\}}$.

Analogously, taking into account that if $F_{0}=L_{1}[0, \infty) \cap L_{\infty}[0, \infty)$ and $F_{1}=L_{1}[0, \infty)+L_{\infty}[0, \infty)$, then

$\left(F_{0}, F_{1}\right)_{[\theta]}=\left\{L_{p}[0, \infty) \cap L_{p^{\prime}}[0, \infty), 1 p=1-\theta, 0<\theta \leq 1 / 2, L_{p}[0, \infty)+L_{p^{\prime}}[0, \infty), 1 p=1-\theta, 1 / 2 \leq \theta<1\right.$,

where $1 / p+1 / p^{\prime}=1$ (see [24]), we obtain for $1<p \leq 2$ (and some $C>0$ ) that

$\widetilde{\beta}_{\mathcal{M}_{n}}\left(T: E_{1} \times \cdots \times E_{n} \rightarrow L_{p}[0, \infty) \cap L_{p^{\prime}}[0, \infty)\right) \leq$

$C \widetilde{\beta}_{\mathcal{M}_{n}}\left(T: E_{1} \times \cdots \times E_{n} \rightarrow L_{1}[0, \infty) \cap L_{\infty}[0, \infty)\right)^{\frac{1}{p}}$

- $\widetilde{\beta}_{\mathcal{M}_{n}}\left(T: E_{1} \times \cdots \times E_{n} \rightarrow L_{1}[0, \infty)+L_{\infty}[0, \infty)\right)^{1-\frac{1}{p}}$, and for $2 \leq p<\infty$ (and

some $C>0)$ that $\widetilde{\beta}_{\mathcal{M}_{n}}\left(T: E_{1} \times \cdots \times E_{n} \rightarrow L_{p}[0, \infty)+L_{p^{\prime}}[0, \infty)\right) \leq$

$C \widetilde{\beta}_{\mathcal{M}_{n}}\left(T: E_{1} \times \cdots \times E_{n} \rightarrow L_{1}[0, \infty) \cap L_{\infty}[0, \infty)\right)^{\frac{1}{p}}$

. $\widetilde{\beta}_{\mathcal{M}_{n}}\left(T: E_{1} \times \cdots \times E_{n} \rightarrow L_{1}[0, \infty)+L_{\infty}[0, \infty)\right)^{1-\frac{1}{p}}$.

The following result can be proved in a similar way to Theorems 4 and 4 . We include the proof for the sake of completeness.

Let $\mathcal{I}_{1}, \ldots, \mathcal{I}_{n}$ be linear operator ideals, let $E_{1}, \ldots, E_{n}$ be Banach spaces and let $\bar{F}=\left(F_{0}, F_{1}\right)$ be a Banach couple. Assume that $F$ is of class $\mathcal{C}_{J}(\theta, \bar{F})$ with constant $C$. For any $T \in \mathcal{L}\left(E_{1}, \ldots, E_{n} ; \Delta(\bar{F})\right)$,

(a) $\beta_{\left[\mathcal{I}_{1}, \ldots, \mathcal{I}_{n}\right]}\left(T: E_{1} \times \cdots \times E_{n} \rightarrow F\right) \leq$ $\left.F_{1}\right)^{\theta}$.$$
C \beta_{\left[\mathcal{I}_{1}, \ldots, \mathcal{I}_{n}\right]}\left(T: E_{1} \times \cdots \times E_{n} \rightarrow F_{0}\right)^{1-\theta} \beta_{\left[\mathcal{I}_{1}, \ldots, \mathcal{I}_{n}\right]}\left(T: E_{1} \times \cdots \times E_{n} \rightarrow\right.
$$

(b) $\beta_{\left[\mathcal{I}_{1}, \ldots, \mathcal{I}_{n}\right]}\left(T: E_{1} \times \cdots \times E_{n} \rightarrow F\right) \leq$ $\Delta(\bar{F}))^{1-\Theta}$,

where $\Theta=\min \{\theta, 1-\theta\}$.

We start by proving (a). Let $\varepsilon_{k}>\beta_{\left[\mathcal{I}_{1}, \ldots, \mathcal{I}_{n}\right]}\left(T: E_{1} \times \cdots \times E_{n} \rightarrow F_{k}\right), k=$ 0,1 . We have that for Banach spaces $Z_{i}^{k}$ and operators $R_{i}^{k} \in \mathcal{I}_{i}\left(E_{i} ; Z_{i}^{k}\right)(i=$ $1, \ldots, n)$, it holds that for all $x_{1} \in E_{1}, \ldots, x_{n} \in E_{n}$

$$
\left\|T\left(x_{1}, \cdots, x_{n}\right)\right\|_{F_{k}} \leq \varepsilon_{k}\left\|x_{1}\right\| \cdots\left\|x_{n}\right\|+\min _{i \in\{1, \ldots, n\}}\left\{\left\|R_{i}^{k}\left(x_{i}\right)\right\|_{Z_{i}^{k}}\left\|x_{1}\right\| \stackrel{[i]}{\cdots}\left\|x_{n}\right\|\right\}, k=0,1 .
$$

We write $Z_{i}$ for $\left(Z_{i}^{0} \oplus Z_{i}^{1}\right)_{1}$ and $\varepsilon:=\min \left\{\varepsilon_{0}, \varepsilon_{1}\right\}$. Take $t>0$ and consider the operator given by $R_{i} x:=\frac{C t^{-\theta} \max \left\{\varepsilon_{0}, t \varepsilon_{1}\right\}}{\varepsilon}\left(R_{i}^{0} x, R_{i}^{1} x\right), x \in E_{i}(i=1, \ldots, n)$. The operators $R_{i}^{0}$ and $R_{i}^{1}$ belong to $\mathcal{I}_{i}^{\varepsilon}$, and so $R_{i}$ also belongs to $\mathcal{I}_{i}(i=1, \ldots, n)$.

By (4) we have, for any $x_{1} \in E_{1}, \ldots, x_{n} \in E_{n},\left\|T\left(x_{1}, \ldots, x_{n}\right)\right\|_{F} \leq C t^{-\theta} J\left(t, T\left(x_{1}, \ldots, x_{n}\right)\right)$

$\leq C t^{-\theta} \max \left\{t^{k}\left(\varepsilon_{k}\left\|x_{1}\right\| \cdots\left\|x_{n}\right\|+\min _{i \in\{1, \ldots, n\}}\left\{\left\|R_{i}^{k}\left(x_{i}\right)\right\|_{Z_{i}^{k}}\left\|x_{1}\right\| \stackrel{[i]}{\cdot{ }^{\prime}}\left\|x_{n}\right\|\right\}\right):\right.$

$k=0,1\}$

$\leq C t^{-\theta} \max \left\{t^{k} \varepsilon_{k}\left(\left\|x_{1}\right\| \cdots\left\|x_{n}\right\|+\frac{1}{\varepsilon} \min _{i \in\{1, \ldots, n\}}\left\{\left\|R_{i}^{k}\left(x_{i}\right)\right\|_{Z_{i}^{k}}\left\|x_{1}\right\| \stackrel{[i]}{\cdots}\left\|x_{n}\right\|\right\}\right):\right.$

$k=0,1\}$ 


$$
\begin{aligned}
& \leq C t^{-\theta} \max \left\{\varepsilon_{0}, t \varepsilon_{1}\right\} \cdot \\
& \cdot \max \left\{\left\|x_{1}\right\| \cdots\left\|x_{n}\right\|+\frac{1}{\varepsilon} \min _{i \in\{1, \ldots, n\}}\left\{\left\|R_{i}^{k}\left(x_{i}\right)\right\|_{Z_{i}^{k}}\left\|x_{1}\right\| \cdot \cdots\left\|x_{n}\right\|\right\}: k=0,1\right\} . \\
& \text { Thus, }\left\|T\left(x_{1}, \ldots, x_{n}\right)\right\|_{F} \leq C t^{-\theta} \max \left\{\varepsilon_{0}, t \varepsilon_{1}\right\} \cdot\left[\left\|x_{1}\right\| \cdots\left\|x_{n}\right\|+\right. \\
& \left.\quad+\frac{1}{\varepsilon} \min _{i \in\{1, \ldots, n\}}\left\{\left(\left\|R_{i}^{0} x_{i}\right\|_{Z_{i}^{0}}+\left\|R_{i}^{1} x_{i}\right\|_{Z_{i}^{1}}\right)\left\|x_{1}\right\| \cdot \cdots\left\|x_{n}\right\|\right\}\right] \\
& =C t^{-\theta} \max \left\{\varepsilon_{0}, t \varepsilon_{1}\right\} \cdot\left[\left\|x_{1}\right\| \cdots\left\|x_{n}\right\|+\right. \\
& \left.\quad+\frac{1}{\varepsilon} \frac{\varepsilon}{C t^{-\theta} \max \left\{\varepsilon_{0}, t \varepsilon_{1}\right\}} \min _{i \in\{1, \ldots, n\}}\left\{\left\|R_{i}\left(x_{i}\right)\right\|_{Z_{i}}\left\|x_{1}\right\| \cdots \cdot\left\|x_{n}\right\|\right\}\right] \\
& =C t^{-\theta} \max \left\{\varepsilon_{0}, t \varepsilon_{1}\right\}\left\|x_{1}\right\| \cdots\left\|x_{n}\right\|+\min _{i \in\{1, \ldots, n\}}\left\{\left\|R_{i}\left(x_{i}\right)\right\| Z_{i}\left\|x_{1}\right\| \cdot \cdots \cdot{ }^{[i]} \cdot x_{n} \|\right\} . \text { So }
\end{aligned}
$$

we obtain

$$
\beta_{\left[\mathcal{I}_{1}, \ldots, \mathcal{I}_{n}\right]}\left(T: E_{1} \times \cdots \times E_{n} \rightarrow F\right) \leq C t^{-\theta} \max \left\{t^{k} \beta_{\left[\mathcal{I}_{1}, \cdots, \mathcal{I}_{n}\right]}\left(T: E_{1} \times \cdots \times E_{n} \rightarrow F_{k}\right): k=0,1\right\},
$$

for any $t>0$. Finally using an analogous reasoning to that used in the last part of the proof of Theorem 4, the estimate given in (a) is proved.

Now we establish (b). Fix $\eta>0$ and let $t \geq 1$ be such that

$$
t^{-\theta} \leq \eta \text { and } t^{\theta-1} \leq \eta
$$

Consider $\sigma>\beta_{\left[\mathcal{I}_{1}, \ldots, \mathcal{I}_{n}\right]}\left(T: E_{1} \times \cdots \times E_{n} \rightarrow \Sigma(\bar{F})\right)$. We can find Banach spaces $H_{i}$ and operators $R_{i} \in \mathcal{I}_{i}\left(E_{i} ; H_{i}\right)(i=1, \ldots, n)$ so that, for all $x_{1} \in E_{1}, \ldots, x_{n} \in$ $E_{n}$,

$$
\left\|T\left(x_{1}, \ldots, x_{n}\right)\right\|_{\Sigma(\bar{F})} \leq \sigma\left\|x_{1}\right\| \cdots\left\|x_{n}\right\|+\min _{i \in\{1, \ldots, n\}}\left\{\left\|R_{i}\left(x_{i}\right)\right\|_{H_{i}}\left\|x_{1}\right\| \stackrel{[i]}{\cdot}\left\|x_{n}\right\|\right\} .
$$

Moreover, if $\delta>\beta_{\left[\mathcal{I}_{1}, \ldots, \mathcal{I}_{n}\right]}\left(T: E_{1} \times \cdots \times E_{n} \rightarrow \Delta(\bar{F})\right)$ then there are Banach spaces $G_{i}$ and operators $S_{i} \in \mathcal{I}_{i}\left(E_{i} ; G_{i}\right)(i=1, \ldots, n)$ for which we have, for $x_{1} \in E_{1}, \ldots, x_{n} \in E_{n}$, that

$$
\left\|T\left(x_{1}, \ldots, x_{n}\right)\right\|_{\Delta(\bar{F})} \leq \delta\left\|x_{1}\right\| \cdots\left\|x_{n}\right\|+\min _{i \in\{1, \ldots, n\}}\left\{\left\|S_{i}\left(x_{i}\right)\right\|_{G_{i}}\left\|x_{1}\right\| \stackrel{[i]}{\cdot}\left\|x_{n}\right\|\right\} .
$$

Now for $\varepsilon>0$ and $x_{1} \in E_{1}, \ldots, x_{n} \in E_{n}, T\left(x_{1}, \ldots, x_{n}\right)$ can be written as $T\left(x_{1}, \ldots, x_{n}\right)=y_{0}+y_{1}, y_{k} \in F_{k}$, and

$$
\left\|y_{k}\right\|_{F_{k}} \leq\left\|y_{0}\right\|_{F_{0}}+\left\|y_{1}\right\|_{F_{1}} \leq(1+\varepsilon)\left\|T\left(x_{1}, \ldots, x_{n}\right)\right\|_{\Sigma(\bar{F})}, \quad k=0,1,
$$

what implies, by (17) and (15), that

$$
\left\|y_{k}\right\|_{F_{k}} \leq(1+\varepsilon) \sigma\left\|x_{1}\right\| \cdots\left\|x_{n}\right\|+(1+\varepsilon) \min _{i \in\{1, \ldots, n\}}\left\{\left\|R_{i}\left(x_{i}\right)\right\|\left\|x_{1}\right\| \stackrel{[i]}{\cdots}\left\|x_{n}\right\|\right\}, k=0,1 .
$$

Taking into account that also $y_{k} \in \Delta(\bar{F})$, and using (17), we get that

$$
\left\|y_{k}\right\|_{F_{1-k}} \leq\left\|T\left(x_{1}, \ldots, x_{n}\right)\right\|_{F_{1-k}}+\left\|y_{1-k}\right\|_{F_{1-k}} \leq(2+\varepsilon)\left\|T\left(x_{1}, \ldots, x_{n}\right)\right\|_{\Delta(\bar{F})}, k=0,1 .
$$


By (16) we have finally, for $k=0,1$,

$$
\left\|y_{k}\right\|_{F_{1-k}} \leq(2+\varepsilon) \delta\left\|x_{1}\right\|_{E_{1}} \cdots\left\|x_{n}\right\|_{E_{n}}+(2+\varepsilon) \min _{i \in\{1, \ldots, n\}}\left\{\left\|S_{i}\left(x_{i}\right)\right\|\left\|x_{1}\right\| \stackrel{[i]}{\cdots}\left\|x_{n}\right\|\right\} .
$$

Therefore, for any $x_{1} \in E_{1}, \ldots, x_{n} \in E_{n}$, we obtain from (4), (14), (18), (19) and the fact that $t \geq 1\left\|T\left(x_{1}, \ldots, x_{n}\right)\right\|_{F} \leq\left\|y_{0}\right\|_{F}+\left\|y_{1}\right\|_{F} \leq C t^{\theta} J\left(t^{-1}, y_{0}\right)+$ $C t^{-\theta} J\left(t, y_{1}\right)$

$\leq C \eta t \max \left\{\left\|y_{0}\right\|_{F_{0}}, t^{-1}\left\|y_{0}\right\|_{F_{1}}\right\}+C \eta \max \left\{\left\|y_{1}\right\|_{F_{0}}, t\left\|y_{1}\right\|_{F_{1}}\right\}$

$\leq C \eta t \max \left\{(1+\varepsilon) \sigma\left\|x_{1}\right\| \cdots\left\|x_{n}\right\|+(1+\varepsilon) \min _{i \in\{1, \ldots, n\}}\left\{\left\|R_{i}\left(x_{i}\right)\right\|_{H_{i}}\left\|x_{1}\right\| \stackrel{[i]}{ } \cdot{ }^{[i]}\right.\right.$ $\left.\left\|x_{n}\right\|\right\}$,

$\left.t^{-1}\left[(2+\varepsilon) \delta\left\|x_{1}\right\| \cdots\left\|x_{n}\right\|+(2+\varepsilon) \min _{i \in\{1, \ldots, n\}}\left\{\left\|S_{i}\left(x_{i}\right)\right\|_{G_{i}}\left\|x_{1}\right\| \stackrel{[i]}{\cdots}\left\|x_{n}\right\|\right\}\right]\right\}$

$+C \eta \max \left\{(2+\varepsilon) \delta\left\|x_{1}\right\| \cdots\left\|x_{n}\right\|+(2+\varepsilon) \min _{i \in\{1, \ldots, n\}}\left\{\left\|S_{i}\left(x_{i}\right)\right\|_{G_{i}}\left\|x_{1}\right\| \stackrel{[i]}{\cdots}\left\|x_{n}\right\|\right\}\right.$,

$\left.t\left[(1+\varepsilon) \sigma\left\|x_{1}\right\| \cdots\left\|x_{n}\right\|+(1+\varepsilon) \min _{i \in\{1, \ldots, n\}}\left\{\left\|R_{i}\left(x_{i}\right)\right\|_{H_{i}}\left\|x_{1}\right\| \stackrel{[i]}{\cdot{ }^{[}}\left\|x_{n}\right\|\right\}\right]\right\}$

$=2 C \eta \max \left\{(1+\varepsilon) \sigma t\left\|x_{1}\right\| \cdots\left\|x_{n}\right\|+(1+\varepsilon) t \min _{i \in\{1, \ldots, n\}}\left\{\left\|R_{i}\left(x_{i}\right)\right\|_{H_{i}}\left\|x_{1}\right\| \cdot{ }^{[i]}\right.\right.$ $\left.\left\|x_{n}\right\|\right\}$,

$\left.(2+\varepsilon) \delta\left\|x_{1}\right\| \cdots\left\|x_{n}\right\|+(2+\varepsilon) \min _{i \in\{1, \ldots, n\}}\left\{\left\|S_{i}\left(x_{i}\right)\right\|_{G_{i}}\left\|x_{1}\right\| \cdot{ }^{[i]} \cdot\left\|x_{n}\right\|\right\}\right\}$

$\leq 2 C \eta \max \{(1+\varepsilon) \sigma t,(2+\varepsilon) \delta\}\left\|x_{1}\right\| \cdots\left\|x_{n}\right\|+$

$+(2+\varepsilon) t \min _{i \in\{1, \ldots, n\}}\left\{\left(\left\|R_{i}\left(x_{i}\right)\right\|_{H_{i}}+\left\|S_{i}\left(x_{i}\right)\right\|_{G_{i}}\right)\left\|x_{1}\right\| \cdots{ }^{[i]}\left\|x_{n}\right\|\right\}$

$=2 C \eta \max \{(1+\varepsilon) \sigma t,(2+\varepsilon) \delta\}\left\|x_{1}\right\| \cdots\left\|x_{n}\right\|+\min _{i \in\{1, \ldots, n\}}\left\{\left\|U_{i}\left(x_{i}\right)\right\|_{V_{i}}\left\|x_{1}\right\| \cdot{ }^{[i]}\right.$ $\left.\left\|x_{n}\right\|\right\}$, where $V_{i}:=\left(H_{i} \oplus G_{i}\right)_{1}$ and $U_{i} x:=(2+\varepsilon) t\left(R_{i} x, S_{i} x\right)$ for all $x \in$ $E_{i}(i=1, \ldots, n)$. Since $R_{i} \in \mathcal{I}_{i}\left(E_{i} ; H_{i}\right)$ and $S_{i} \in \mathcal{I}_{i}\left(E_{i} ; G_{i}\right)$, it follows that $U_{i} \in \mathcal{I}_{i}\left(E_{i} ; V_{i}\right)$ for every $i=1, \ldots, n$, and then

$$
\beta_{\left[\mathcal{I}_{1}, \ldots, \mathcal{I}_{n}\right]}\left(T: E_{1} \times \cdots \times E_{n} \rightarrow F\right) \leq 2 C \eta \max \{(1+\varepsilon) \sigma t,(2+\varepsilon) \delta\} .
$$

Whence $\beta_{\left[\mathcal{I}_{1}, \ldots, \mathcal{I}_{n}\right]}\left(T: E_{1} \times \cdots \times E_{n} \rightarrow F\right) \leq 2 C \eta$.

$\cdot \max \left\{\beta_{\left[\mathcal{I}_{1}, \ldots, \mathcal{I}_{n}\right]}\left(T: E_{1} \times \cdots \times E_{n} \rightarrow \Sigma(\bar{F})\right) t, 2 \beta_{\left[\mathcal{I}_{1}, \ldots, \mathcal{I}_{n}\right]}\left(T: E_{1} \times \cdots \times E_{n} \rightarrow\right.\right.$ $\Delta(\bar{F}))\}$. Now similar arguments to those used in the final part of the proof of Theorem 4 allow to establish the validity of (b).

Using Theorem 4, analogous estimates to those obtained just before that theorem for $\widetilde{\beta}_{\mathcal{M}_{n}}$ also hold for the measure $\beta_{\left[\mathcal{I}_{1}, \ldots, \mathcal{I}_{n}\right]}$.

\section{Some examples and applications related to sum- ming operators}

It is well-known that the notion of summing operator can be generalized in different ways to the multilinear setting, and each of them has shown to be useful depending on the particular application. We will center our attention in generalizations that allow to get factorization theorems for the corresponding 
multilinear map. These are mainly variants of dominated multilinear operators and factorable summing multilinear operators.

\subsection{Examples of multilinear operators belonging to the closed injective hull of summing multilinear operators}

Recall that $T \in \mathcal{L}(E ; F)$ is absolutely summing if there exists $K>0$ such that

$$
\sum_{k=1}^{n}\left\|T\left(x_{k}\right)\right\| \leq K \sup _{x^{*} \in B_{E^{*}}}\left(\sum_{k=1}^{n}\left|\left\langle x_{k}, x^{*}\right\rangle\right|\right)
$$

for every finitely many $x_{1}, \ldots, x_{n} \in E$. The set of all absolutely summing linear operators, which is denoted by $\Pi_{1}$, is an injective Banach linear operator ideal (see for instance [20, Theorem 19.5.3] or [15, Chapter I, Section 11]). We will use the following useful characterization [20, Corollary 20.7.5] (see also [30, Theorem 17.3.2]):

The following assertions are equivalent for any $T \in \mathcal{L}(E ; F)$.

(a) $T \in{\overline{\Pi_{1}}}^{i n j}(E ; F)$.

(b) There is a function $N: R^{+} \rightarrow R^{+}$and a regular Borel probability measure $\eta$ on $B_{E^{*}}$ such that

$$
\|T(x)\| \leq N(\varepsilon) \int_{B_{E^{*}}}\left|\left\langle x, x^{*}\right\rangle\right| d \eta\left(x^{*}\right)+\varepsilon\|x\|, \text { for each } \varepsilon>0 \text { and } x \in E .
$$

The characterization given by Lemma 5.1 allows to define a measure associated to the ideal of absolutely summing operators as follows: given $T \in \mathcal{L}(E ; F)$, consider the function

$\beta_{\mathcal{N}}(T):=\inf \left\{\varepsilon>0:\|T(x)\| \leq N(\varepsilon) \int_{B_{E^{*}}}\left|\left\langle x, x^{*}\right\rangle\right| d \eta\left(x^{*}\right)+\varepsilon\|x\|, x \in E\right.$, for a given function

$$
\left.N: R^{+} \rightarrow R^{+} \text {and a given regular Borel probability measure } \eta \text { both depending only on } T\right\} \text {. }
$$

Consequently, $T$ belongs to $\bar{\Pi}_{1}^{i n j}$ if and only $\beta_{\mathcal{N}}(T)=0$.

Jarchow and Matter [21] considered that concrete choices of the function $N$ in Lemma 5.1(b) provide better descriptions of classes of operators that are included in the closed injective hull of the ideal of summing operators. For any $0<\sigma<1$ and $K>0$ let $r=\sigma /(1-\sigma)$ and define $N_{1}(\varepsilon)=\frac{K}{\varepsilon^{r}}$. It is not hard to check that (20) is equivalent to (see [21, p.47] and also [26, p.195]) $\|T(x)\| \leq$ $K\|x\|^{\sigma}\left(\int_{B_{E^{*}}}\left|\left\langle x, x^{*}\right\rangle\right| d \eta\left(x^{*}\right)\right)^{1-\sigma}$, forall $\mathrm{x} \in E$. This last inequality defines, for $0 \leq \sigma<1$, the class of $(1, \sigma)$-absolutely continuous operators. Therefore, the class of $(1, \sigma)$-absolutely continuous operators, that trivially contains the class $\Pi_{1}$ of all absolutely summing operators, is actually contained in its closed 
injective hull ${\overline{\Pi_{1}}}^{i n j}$. A similar treatment can be done for arbitrary $1 \leq p<\infty$. The class $\Pi_{(p, \sigma)}$ is formed by all $(p, \sigma)$-absolutely continuous operators, that is, all $T \in \mathcal{L}(E ; F)$ for which there exist a constant $K>0$ and a regular Borel probability measure $\eta$ such that

$$
\|T(x)\| \leq K\|x\|^{\sigma}\left(\int_{B_{E^{*}}}\left|\left\langle x, x^{*}\right\rangle\right|^{p} d \eta\left(x^{*}\right)\right)^{(1-\sigma) / p}, \text { forany } \in E .
$$

In this general case, Lemma 5.1 reads as follows (see [20, Corollary 20.7.5]): $T$ belongs to $\bar{\Pi}_{p}^{i n j}(E ; F)$ if and only if there is a function $N: R^{+} \rightarrow R^{+}$and a regular Borel probability measure $\eta$ on $B_{E^{*}}$ such that

$$
\|T(x)\| \leq N(\varepsilon)\left(\int_{B_{E^{*}}}\left|\left\langle x, x^{*}\right\rangle\right|^{p} d \eta\left(x^{*}\right)\right)^{1 / p}+\varepsilon\|x\|, \text { foreach }
$$

$\varepsilon>0$ and $x \in E$.

\section{Replacing}

$\mathrm{N}(\varepsilon)$ with a suitable $N_{1}(\varepsilon)$ and doing similar calculations as for $p=1$, we get that $\Pi_{(p, \sigma)} \subset{\overline{\Pi_{p}}}^{i n j}$.

Now we use this information in the multilinear case. Take $1 \leq p \leq p_{1}, \ldots, p_{n}<$ $\infty$ such that $1 / p=\sum_{i=1}^{n} 1 / p_{i}$ and $0 \leq \sigma<1$. According [14, Theorem 3.3], an $n$ linear operator $T \in \mathcal{L}\left(E_{1}, \ldots, E_{n} ; F\right)$ is $\left(p ; p_{1}, \ldots, p_{n} ; \sigma\right)$-absolutely continuous (in symbols $\left.T \in \mathcal{L}_{a s,\left(p ; p_{1}, \cdots, p_{n}\right)}^{\sigma}\right)$ if there are regular Borel probability measures $\mu_{1}, \ldots, \mu_{n}$ on $B_{E_{1}^{*}}, \ldots, B_{E_{n}^{*}}$, respectively, and a constant $K>0$, in such a way that for every $x_{1} \in E_{1}, \ldots, x_{n} \in E_{n}$,

$$
\left\|T\left(x_{1}, \ldots, x_{n}\right)\right\| \leq K \prod_{i=1}^{n}\left\|x_{i}\right\|^{\sigma}\left(\int_{B_{E_{i}^{*}}}\left|\left\langle x_{i}, x_{i}^{*}\right\rangle\right|^{p_{i}} d \mu_{i}\left(x_{i}^{*}\right)\right)^{(1-\sigma) / p_{i}} .
$$

The infimum of all $K>0$ is the norm $\|T\|_{\mathcal{L}_{a s,\left(p ; p_{1}, \ldots, p_{n}\right)}^{\sigma}}$. The linear case gives directly the inclusion:

$$
\mathcal{L}_{a s,\left(p ; p_{1}, \ldots, p_{n}\right)}^{\sigma} \subset\left[\bar{\Pi}_{p_{1}}^{i n j}, \ldots, \bar{\Pi}_{p_{n}}^{i n j}\right] .
$$

Therefore, for every $T \in \mathcal{L}_{a s,\left(p ; p_{1}, \ldots, p_{n}\right)}^{\sigma}$, it holds that

$$
\beta_{\left[\Pi_{p_{1}}, \ldots, \Pi_{p_{n}}\right]}(T)=\widetilde{\beta}_{\left[\bar{\Pi}_{p_{1}}^{i n j}, \ldots, \bar{\Pi}_{p_{n}}^{i n j}\right]}(T)=0 .
$$

Just as some examples, let us apply these ideas to get new classes of operators contained in $\bar{\Pi}_{1}{ }^{i n j}$.

(a) We start by considering a function $N_{0}$ such that $N_{0}<N_{1}$. Then, when replacing $N$ with $N_{0}$ in Lemma 5.1(b), we obtain a new class of operators $\Pi_{N_{0}}$ that is contained in $\Pi_{(1, \sigma)}$ for all $0<\sigma<1$. This is the case if we take, for instance, $N_{0}(\varepsilon)=K \log \left(\frac{1}{\varepsilon}\right)$, as we next show. Fix $x \in E$. The function $\phi_{0}(\varepsilon)=N_{0}(\varepsilon) \int_{B_{E^{*}}}\left|\left\langle x, x^{*}\right\rangle\right| d \eta\left(x^{*}\right)+\varepsilon\|x\|$ has a minimum at

$$
\varepsilon_{x}:=\frac{K \int_{B_{E^{*}}}\left|\left\langle x, x^{*}\right\rangle\right| d \eta\left(x^{*}\right)}{\|x\|}
$$


and

$$
\phi_{0}\left(\varepsilon_{x}\right)=K\left(\log \frac{\|x\|}{K \int_{B_{E^{*}}}\left|\left\langle x, x^{*}\right\rangle\right| d \eta\left(x^{*}\right)}+1\right) \int_{B_{E^{*}}}\left|\left\langle x, x^{*}\right\rangle\right| d \eta\left(x^{*}\right) .
$$

Note that the above holds for arbitrary $x \in E$ with $\|T(x)\| \neq 0$. Therefore, the new class $\Pi_{N_{0}}$ is defined by all $T \in \mathcal{L}(E ; F)$ for which there is a probability measure $\eta$ such that

$$
\|T(x)\| \leq K\left(\log \frac{\|x\|}{K \int_{B_{E^{*}}}\left|\left\langle x, x^{*}\right\rangle\right| d \eta\left(x^{*}\right)}+1\right) \int_{B_{E^{*}}}\left|\left\langle x, x^{*}\right\rangle\right| d \eta\left(x^{*}\right)
$$

for all $x \in E$ with $\|T(x)\| \neq 0$. Note that this domination shows in particular that $\Pi_{1} \subset \Pi_{N_{0}}$.

(b) If we consider a function $N_{2}$ with $N_{1}<N_{2}$, then we get a new class of linear operators that contains $\Pi_{(1, \sigma)}$ for all $0<\sigma<1$ but it is still contained in ${\overline{\Pi_{1}}}^{i n j}$. For instance, take $N_{2}(\varepsilon)=K e^{\frac{1}{\varepsilon}}$. Fix $x \in E$ with $T(x) \neq 0$. In this case, it is not possible to give an explicit formula for the point $\varepsilon_{x}$ where the function $\phi_{2}(\varepsilon)=N_{2}(\varepsilon) \int_{B_{E^{*}}}\left|\left\langle x, x^{*}\right\rangle\right| d \eta\left(x^{*}\right)+\varepsilon\|x\|$ attains its minimum, as this point is given by the solution of the equation

$$
K e^{1 / \varepsilon_{x}} \int_{B_{E^{*}}}\left|\left\langle x, x^{*}\right\rangle\right| d \eta\left(x^{*}\right)=\varepsilon_{x}^{2}\|x\| .
$$

The next example refers to the multilinear case.

(a) Using arguments as in the second part of the proof of Theorem 3 and taking into account Lemma 5.1, we obtain the following: Let $E_{1}, \ldots, E_{n}$ be Banach spaces and let $T \in \mathcal{L}\left(E_{1}, \ldots, E_{n} ; F\right)$. If there are functions $N_{i}: R^{+} \rightarrow$ $R^{+}$and regular Borel probability measures $\eta_{i}$ on $B_{E_{i}^{*}}(i=1, \ldots, n)$ such that $\left\|T\left(x_{1}, \ldots, x_{n}\right)\right\| \leq\left(N_{1}\left(\varepsilon_{1}\right) \int_{B_{E_{1}^{*}}}\left|\left\langle x_{1}, x_{1}^{*}\right\rangle\right| d \eta_{1}\left(x_{1}^{*}\right)+\varepsilon_{1}\left\|x_{1}\right\|\right) \ldots$ $\cdots\left(N_{n}\left(\varepsilon_{n}\right) \int_{B_{E_{n}^{*}}}\left|\left\langle x_{n}, x_{n}^{*}\right\rangle\right| d \eta_{n}\left(x_{n}^{*}\right)+\varepsilon_{n}\left\|x_{n}\right\|\right)$, for all $\varepsilon_{1}>0, \ldots, \varepsilon_{n}>0$ and $x_{1} \in E_{1}, \ldots, x_{n} \in E_{n}$, then $T \in\left[{\overline{\Pi_{1}}}^{i n j}, \ldots, \bar{\Pi}_{1}^{i n j}\right]\left(E_{1}, \ldots, E_{n} ; F\right)$.

(b) When we now take, for instance, the functions $N_{i}$ as the function $N_{0}$ considered in Example 5.1(a) we deduce in particular: Let $E_{1}, \ldots, E_{n}$ be Banach spaces and let $T \in \mathcal{L}\left(E_{1}, \ldots, E_{n} ; F\right)$. If there are constants $K_{1}>0, \ldots, K_{n}>0$ and regular Borel probability measures $\eta_{1}, \ldots, \eta_{n}$ on $B_{E_{1}^{*}}, \ldots, B_{E_{n}^{*}}$, respectively, such that $\left\|T\left(x_{1}, \ldots, x_{n}\right)\right\| \leq K_{1}\left(\log \frac{\left\|x_{1}\right\|_{E_{1}}}{K_{1} \int_{B_{E_{1}^{*}}^{*}}\left|\left\langle x_{1}, x_{1}^{*}\right\rangle\right| d \eta_{1}\left(x_{1}^{*}\right)}+1\right) \int_{B_{E_{1}^{*}}}\left|\left\langle x_{1}, x_{1}^{*}\right\rangle\right| d \eta_{1}\left(x_{1}^{*}\right) \cdots$ $\cdots K_{n}\left(\log \frac{\left\|x_{n}\right\|_{E_{n}}}{K_{n} \int_{B_{E_{n}^{*}}}\left|\left\langle x_{n}, x_{n}^{*}\right\rangle\right| d \eta_{n}\left(x_{n}^{*}\right)}+1\right) \int_{B_{E_{n}^{*}}}\left|\left\langle x_{n}, x_{n}^{*}\right\rangle\right| d \eta_{n}\left(x_{n}^{*}\right)$, for all $x_{1} \in E_{1}, \ldots, x_{n} \in$ $E_{n}$, then $T \in\left[{\overline{\Pi_{1}}}^{i n j}, \ldots,{\overline{\Pi_{1}}}^{i n j}\right]\left(E_{1}, \ldots, E_{n} ; F\right)$. 


\subsection{Interpolation and closed injective hull of summing mul- tilinear operators}

The application of the corresponding interpolation formulas obtained in Section 4 allows to relate some classes of multilinear operators considered in Section 5. Let us finish the paper by showing a concrete example of this, concerning interpolation and multilinear operators belonging to $\mathcal{L}_{a s,\left(p ; p_{1}, \ldots, p_{n}\right)}^{\sigma}$ with values in Lorentz spaces. We will use (6). Direct consequences of Theorem 4 and Theorem 3 are the following results.

Let $\mathcal{I}_{1}, \ldots, \mathcal{I}_{n}$ be linear operator ideals and let $E_{1}, \ldots, E_{n}$ be Banach spaces. Suppose that $T \in \mathcal{L}\left(E_{1}, \ldots, E_{n} ; L_{\infty} \cap L_{1}\right)$. For $0<\theta=1 / p<1,1 \leq q \leq \infty$, there is $C>0$ such that $\beta_{\left[\mathcal{I}_{1}, \ldots, \mathcal{I}_{n}\right]}\left(T: E_{1} \times \cdots \times E_{n} \rightarrow L_{p, q}\right) \leq$ $C \beta_{\left[\mathcal{I}_{1}, \ldots, \mathcal{I}_{n}\right]}\left(T: E_{1} \times \cdots \times E_{n} \rightarrow L_{\infty}\right)^{1-\theta} \beta_{\left[\mathcal{I}_{1}, \ldots, \mathcal{I}_{n}\right]}\left(T: E_{1} \times \cdots \times E_{n} \rightarrow L_{1}\right)^{\theta}$.

Let $1 / p=1 / p_{1}+\cdots+1 / p_{n}, 1<p, p_{i}<\infty, \theta=1 / p$ and $1 \leq q \leq \infty$. Let $T \in \mathcal{L}\left(E_{1}, \ldots, E_{n} ; L_{\infty} \cap L_{1}\right)$, with $T \in \mathcal{L}_{a s,\left(p ; p_{1}, \ldots, p_{n}\right)}^{\sigma}\left(E_{1}, \ldots, E_{n} ; L_{\infty}\right)$ or $T \in$ $\mathcal{L}_{a s,\left(p ; p_{1}, \ldots, p_{n}\right)}^{\sigma}\left(E_{1}, \ldots, E_{n} ; L_{1}\right)$. Then $T \in\left[\bar{\Pi}_{p_{1}}^{i n j}, \ldots, \bar{\Pi}_{p_{n}}^{i n j}\right]\left(E_{1}, \ldots, E_{n} ; L_{p, q}\right)$. If for example $T \in \mathcal{L}_{a s,\left(p ; p_{1}, \ldots, p_{n}\right)}^{\sigma}\left(E_{1}, \ldots, E_{n} ; L_{1}\right)$, then $T \in\left[\bar{\Pi}_{p_{1}}^{i n j}, \ldots, \bar{\Pi}_{p_{n}}^{i n j}\right]\left(E_{1}, \ldots, E_{n} ; L_{1}\right)$. By Theorem 3,

$$
\beta_{\left[\Pi_{p_{1}}, \ldots, \Pi_{\left.p_{n}\right]}\right]}\left(T: E_{1} \times \cdots \times E_{n} \rightarrow L_{1}\right)=0 .
$$

It follows from Corollary 5.2 that

$$
\beta_{\left[\Pi_{p_{1}}, \ldots, \Pi_{p_{n}}\right]}\left(T: E_{1} \times \cdots \times E_{n} \rightarrow L_{p, q}\right)=0,
$$

and so, again by Theorem 3, we get the result.

Acknowledgements:

The authors would like to thank the referees for their comments which have led to improve the paper.

A. Manzano was supported in part by the Ministerio de Economía, Industria y Competitividad and FEDER under project MTM2017-84058-P.

P. Rueda and E. A. Sánchez-Pérez were supported in part by the Ministerio de Economía, Industria y Competitividad and FEDER under project MTM201677054-C2-1-P.

\section{References}

[1] R. Arens, The adjoint of a bilinear operation, Proc. Amer. Math. Soc. 2, 839-848 (1951).

[2] R. M. Aron, and P. D. Berner, A Hahn-Banach extension theorem for analytic mappings, Bull. Soc. Math. France 106, 3-24 (1978).

[3] R. M. Aron, B. J. Cole, and T. W. Gamelin, Weak-star continuous analytic functions, Canadian J. Math. 47, 673-683 (1995). 
[4] K. Astala, On measures of non-compactness and ideal variations in Banach spaces, Ann. Acad. Sci. Fenn. Ser. A. I. Math. Dissertationes 29, 1-42 (1980).

[5] J. Bergh, and J.Löfström, Interpolation Spaces (An Introduction) (Springer-Verlag, Berlin, 1976).

[6] O. J. Beucher, On interpolation of strictly (co-)singular linear operators, Proc. Royal Soc. Edinburgh, Section A Mathematics 112A, 263-269 (1989).

[7] G. Botelho, Weakly compact and absolutely summing polynomials, J. Math. Anal. Appl. 265, 458-462 (2002).

[8] G. Botelho, P. Galindo, and L. Pellegrini, Uniform approximation on ideals of multilinear mappings, Math. Scand. 106, 301-319 (2010).

[9] G. Botelho, D. Pellegrino, and P. Rueda, On composition ideals of multilinear mappings and homogeneous polynomials, Publ. Res. Inst. Math. Sci. 43, 1139-1155 (2007).

[10] H. A. Braunss, and H. Junek, Factorization of injective ideals by interpolation, J. Math. Anal. Appl. 297, 740-750 (2004).

[11] F. Cobos, A. Manzano, and A. Martínez, Interpolation theory and measures related to operator ideals, Quart. J. Math. 50, 401-416 (1999).

[12] F. Cobos, A. Manzano, A. Martínez, and P. Matos, On interpolation of strictly singular operators, strictly co-singular operators and related operator ideals, Proc. Royal Soc. Edinburgh, Section A Mathematics 130A, 971-989 (2000).

[13] F. Cobos, and A. Martínez, Remarks on interpolation properties of the measure of weak non-compactness and ideal variations, Math. Nachr. 208, 93-100 (1999).

[14] E. Dahia, D. Achour, and E. A. Sánchez-Pérez, Absolutely continuous multilinear operators, J. Math. Anal. Appl. 397, 205-224 (2013).

[15] A. Defant, and K. Floret, Tensor Norms and Operator Ideals (NorthHoland, Amsterdam, 1993).

[16] M. González, and J. M. Gutiérrez, Injective factorization of holomorphic mappings, Proc. Amer. Math. Soc. 127, 1715-1721 (1999).

[17] M. González, and J. M. Gutiérrez, Erratum to "Injective factorization of holomorphic mappings", Proc. Amer. Math. Soc. 129, 1255-1256 (2001).

[18] A. Grothendieck, Sur certains sous-espaces vectoriels de $L^{p}$, Canadian J. Math. 1, 158-160 (1954). 
[19] S. Heinrich, Closed operator ideals and interpolation, J. Funct. Anal. 35, 397-411 (1980).

[20] H. Jarchow, Locally Convex Spaces (Teubner, Stuttgar, 1981).

[21] H. Jarchow, and U. Matter, Interpolative constructions for operator ideals, Note Mat. 8, 45-56 (1988).

[22] T. Kato, Perturbation theory for nulity, deficiency and other quantities of linear operators, J. D'Analyse Math. 6, 261-322 (1958).

[23] J. A. López Molina, and E. A. Sánchez Pérez, On operator ideals related to $(p, \sigma)$-absolutely continuous operators, Studia Math. 138, 25-40 (2000).

[24] L. Maligranda, Interpolation between sum and intersection of Banach spaces, J. Approx. Theory 47, 42-53 (1986).

[25] L. Maligranda, and A. Quevedo, Interpolation of weakly compact operators, Arch. Math. 55, 280-284 (1990).

[26] U. Matter, Absolutely Continuous Operators and Super Reflexivity, Math. Nach. 130, 193-216 (1987).

[27] A. Pełczyński, On weakly compact polynomial operators on B-spaces with Dunford-Pettis property, Bull. Acad. Polon. Sci. Sér. Sci. Math. Astronom. Phys. 11, 371-378 (1963).

[28] D. Pellegrino, P. Rueda, and E. A. Sánchez-Pérez, Surveying the spirit of absolute summability on multilinear operators and homogeneous polynomials, Rev. R. Acad. Cienc. Exactas Fís. Nat. Ser. A Math. RACSAM 110, 285-302 (2016).

[29] D. Pellegrino, P. Rueda, and E. A. Sánchez-Pérez, Improving integrability via absolute summability: a general version of Diestel's Theorem, Positivity 20, 369-383 (2016).

[30] A. Pietsch, Operator Ideals (North-Holland, Amsterdam, 1980).

[31] A. Pietsch, Ideals of multilinear functionals (designs of a theory), in: Proc. Second Int. Conf. on Operator Algebras, Ideals and Their Applications in Theoretical Physics, Teubner-Texte Math. 67 (Teubner, Leipzig, 1983), $185-199$.

[32] D. Popa, A note on the concept of factorable strongly p-summing operators, Rev. R. Acad. Cienc. Exactas Fís. Nat. Ser. A Math. RACSAM 111, 465471 (2017).

[33] H. Triebel, Interpolation Theory, Function Spaces, Differential Operators (North-Holland, Amsterdam, 1978).

[34] H. O. Tylli, The essential norm of an operator is not self-dual, Israel J. Math. 91, 93-110 (1995). 